\title{
An Instrumented Steering Wheel for
}

\section{Driver Model Development}

\author{
Massimiliano Gobbi ${ }^{\mathrm{a}}$, Francesco Comollia, Masatoshi Hada ${ }^{\mathrm{b}}$, Gianpiero Mastinu ${ }^{\mathrm{a}^{*}}$
}

\begin{abstract}
a Department of Mechanical Engineering, Politecnico di Milano (Technical University), Via La Masa 1, 20156, Milan, Italy.E-mail addresses: massimiliano.gobbi@polimi.it, francesco.comolli@polimi.it

${ }^{b}$ Vehicle Dynamics Laboratory TOYOTA Central R\&D Labs., Inc., 41-1, Yokomichi, Nagakute, Aichi 480-1192, Japan. E-mail address: masatoshi-hada@mosk.tytlabs.co.jp
\end{abstract}

* Corresponding author: Tel. +39 022399 8289, e-mail address: gianpiero.mastinu@polimi.it

\section{Abstract}

The paper focuses on the development and first employment of a new Instrumented Steering Wheel (ISW), useful to conceive future controls for Advanced Driver Assistance Systems or Automated Vehicles. The Instrumented Steering Wheel is particularly suited to develop new and accurate driver models, which are needed to enable the addressed controls. By the new Instrumented Steering wheel, the effects on vehicle control of the Neuro-Muscular System of drivers can be studied.

The ISW senses, at each hand, three forces and three moments. Additionally, the grip at each of the two hands is sensed. The design of the ISW has been performed based on advanced modelling and optimization techniques. The mass, moments of inertia and structural stiffness of the ISW are the ones of a common steering wheel. Both dynamic and static tests of the ISW have been performed to validate the design models and to assess the measurement characteristics. The high accuracy of the ISW is obtained after compensation of inertial effects induced either by gravity, or ISW rotation angle or vehicle motion.

An early employment of the ISW has been made. During an emergency manoeuvre on a test track, the role of each hand in applying the total moment at the steering wheel has been highlighted. The hands apply at the steering wheel not only forces but also extremely relevant moments. The Neuro-Muscular Systems of the two arms, two wrists and two hands are activated separately and according with a pattern that deserve further study. This observation provides a comprehensive basis for the future development of new accurate driver models.

Keywords: Steering Wheel, ADAS, Automated Vehicles, Driver model, Load cell, Neuro-Muscular System activation.

\section{Introduction}

Designing the controls of either Advanced Driver Assistance Systems (ADAS) or Automated Vehicles (AV) requires an accurate driver model [1]. Ten groups of ADAS were identified in [2] and five groups of ADAS were considered in [3]. The design of all such ADAS systems could benefit from a driver model in which the activation of the Neuro-Muscular System (NMS) is properly taken into account. Actually, the delay between the warning provided by ADAS and the driver's activation of the NMS takes a crucial role for the success of the driving manoeuvre. The driver's reaction time depends mostly on the activation of the NMS. We know

Abbreviations: ISW - Instrumented Steering Wheel, ADAS - Advanced Driver Assistance Systems, AV - Automated Vehicles, NMS - Neuro-Muscular System, EPS - Electronic Power Steering, EMG - Electromyography, CAN - Controlled Area Network, FEM Finite Element Model, CFRP - Carbon Fibre Reinforced Polymer, DSP - Digital Signal Processor, CoG - Centre of Gravity. 
(combining the results from references reported in [4] and [5]) that the visual perception by the retina requires approximately $50-100 \mathrm{~ms}$, the brain processing of the different external stimuli requires, to make a decision, approximately $100 \mathrm{~ms}$. The activation of the NMS requires 300 to $800 \mathrm{~ms}$. The delay of actuation of the vehicle systems (steering column, electric- or hydraulic power steering, tyres, ...) requires some more time for the manoeuvre to be completed. In case of a steering action, up to some hundred milliseconds may be necessary, depending on the manoeuvre [1,5]. It appears that the NMS activation provides the biggest delay in the overall driving manoeuvre, i.e. the driver's reaction time depends mainly on the NMS activation. Unfortunately, at the moment, despite the many attempts reported in [1,5], a generally accepted driver model seems not being available, especially for what concerns the accurate modelling of the driver's reaction time.

From the addressed analysis of time delays, we see that the activation of the NMS takes a crucial role within the total driver's reaction time. A pioneering and fundamental contribution for including the NMS activation into a driver model has been made by Cole et Al. [6-10]. In [6], a comprehensive review is given on how to include the NMS activation into a driver model. In [6-10] the driver model with NMS activation provides the total moment at the steering wheel. The single forces and moments applied by the two hands (arms) on the steering wheel are combined into a total moment acting on the steering column. It appears that a driver model with separate NMS activation for the two arms and hands could, in principle, provide a better estimation of the driver's reaction time. Focusing on an accurate NMS activation of hands and arms may guarantee an accurate estimation of driver's reaction time. A study in such a direction is still to be fully deployed, focusing on moments applied by each hand at the steering wheel $[7,16]$. An ISW able to measure separately the forces and moments applied by each hand (arm) seems necessary for the NMS activation analysis.

This introduction section has started focusing on ADAS, because the conceptual design of AV controls is still under development. The demand of an accurate driver model for deriving effective AV controls is even more urgent than for ADAS.

The paper will not deal with the derivation of controls of ADAS or AV but will focus on an Instrumented Steering Wheel that enables the derivation of accurate driver models, to be included into mentioned controls. Such controls, providing the proper assistance during the time instants in which the driving control is passed or shared, require an in-depth knowledge of the interaction between the driver and the steering system.

In the literature, some sensors exist that are similar to the ISW introduced in this paper. In [7], the neuromuscular activity of the human arms while steering is studied, and a test bench with a six-axis load cell mounted on the steering column has been developed. The forces applied by each hand are then estimated with some error, generated by the assumption of no moment applied by each single hand. Forces and moments (torques) at each hand can be measured by the ISW presented in this paper. The ISW could be used in combination with electromyography (EMG) signals to relate them to the forces, moments and grip applied by each hand [8-9]. A similar force platform has been adopted in [11] to compare some new steering wheel designs.

There are many commercial ISWs able to measure the total steering moment [12-15], no distinction is provided whether the moment comes from one hand or the other. Many of such ISWs measure the steady resultant moment at the steering wheel with reasonable performance. However, measuring accurately the transient steering moment could be hard to be achieved by current commercial ISWs. Actually, often, for some commercial ISWs, the moment of inertia around the rotation axis of the steering wheel is not equal to the one of a reference/common steering wheel.

In the literature, an ISW is presented which measures the forces at each hand in three separate sections, without any information of the grip force [16]. In [16], the authors use the ISW on a static driving simulator

[Digitare qui] 
in a lane tracking test. Since the position of the hand is not detected properly, the derivation of moments id difficult. Another ISW, which measures forces and moments, has been presented in [17]. The performance and accuracy of the ISW presented in this paper have been improved with respect to the ones reported in [17].

The new ISW aims to provide, for each single hand, very accurate forces, moments and grip forces, better than in current applications.

The detection of the moment at the steering wheel is useful in the development of control logics for Electronic Power Steering (EPS) systems, as shown in [18] where a device is used to derive the steering moment. We can state that measuring the steering moment is a relevant information, we propose to measure not only the resultant steering moment but the action of each hand. This can be important to let EPS cope with moment activation patterns, still to be discovered and studied [1].

An important application of the measurement of steering wheel forces is the tuning of EPS logics for particular categories of users, like elderly people or people affected by certain pathologies [19]. In this case it is important to acquire the forces and the moments at each hand [16]. In [16], the Authors suggest that having an instrumented steering wheel is useful in the calibration of electromyography (EMG) signals during driving, as done in [7]. Being our new ISW particularly accurate, it could be used for improved applications like the ones mentioned.

The detection of the driver fatigue level is another interesting application for the ISW. Generally, only grip forces are detected for such investigations. In [20-24] some examples have been presented. In [25-28] the perception of the vibrations by the driver has been studied. The accuracy seems different with respect to the one offered by the new ISW.

The paper is organized as follows. In Section 2 the motivations referring on why a NMS activation model should be studied by means of an accurate IWS are given. In Section 3, the concept design of the new ISW is introduced. In Section 4, the ISW load cells are presented. In Section 5, the carbon fibres composite steering wheel body is designed. In Section 6, the data acquisition system is described starting from the voltage output measure from the strain gauges, through the signal processing, to the CAN (Controlled Area Network) data publication. In Section 7 the load cell calibration process is described. That calibration matrix is compared with the calibration matrix computed by FEM (Finite Element Model). In Section 8, the error analysis is performed and the measured performance are evaluated in terms of accuracy and linearity. In Section 9, an experimental natural frequency analysis using accelerometers attached to the steering wheel is presented. Finally, Section 10 describes how the ISW can be used on a test track, with real data acquisition, useful to cast new driver models based on NMS activation. Section 11 discusses critically the aims and limitations of the presented research.

\section{Motivation}

In this Section, the motivation on why an ISW should be used to derive accurate driver models for developing effective controls for ADAS or AV is provided.

The starting point is the contribution given in [6-10] where the activation of the Neuro-Muscular System (NMS) has been included into a driver model. Most of the driver's reaction time is due to the activation of the NMS. In [6], a driver model is presented which generates a moment at the steering wheel of a vehicle. In [6], it appears that the activation of the NMS is not differentiated for the two hands (arms). The control loop of the driver model introduced in [6] is re-arranged in Fig. 1. As the human has perceived and interpreted the external signals (perception and brain processing) a so-called co-activation of neurons is performed. Both alpha- and gamma-neurons are activated. Gamma-neurons activate the muscle spindle which acts as a kind

[Digitare qui] 
of internal sensor for the muscle and perceives the change of the length of the muscle. The alpha-neurons command the contraction of muscle. The muscles apply a moment to the steering wheel. The total moment on the steering wheel is due to the moment applied by muscles, to the intrinsic muscle stiffness and damping, to the inertia of the arms and to moment feedback from the steering column. The steering wheel angle can be computed starting from the total moment applied at the steering wheel. The muscles can contract due to voluntary activation or by reflex. In Fig. 1 the reflex loop is highlighted which depends on the steering wheel angle. Fig. 1 refers to one single NMS activation block. It refers to a set of muscles that are activated contemporarily. In the actual case, however, two arms with hands are applying forces and moments on the steering wheel. A number of muscles are activated (co-contraction) with a pattern that, at the moment, is known insufficiently. Hands and arms apply not only forces but moments (torques) in a full spatial arrangement. The complexity of human muscle system may require several different muscle activation blocks, as shown in Fig. 2.



Fig. 1 -Driver-vehicle model and activation of the Neuro-Muscular System, re-arranged from [6]. 


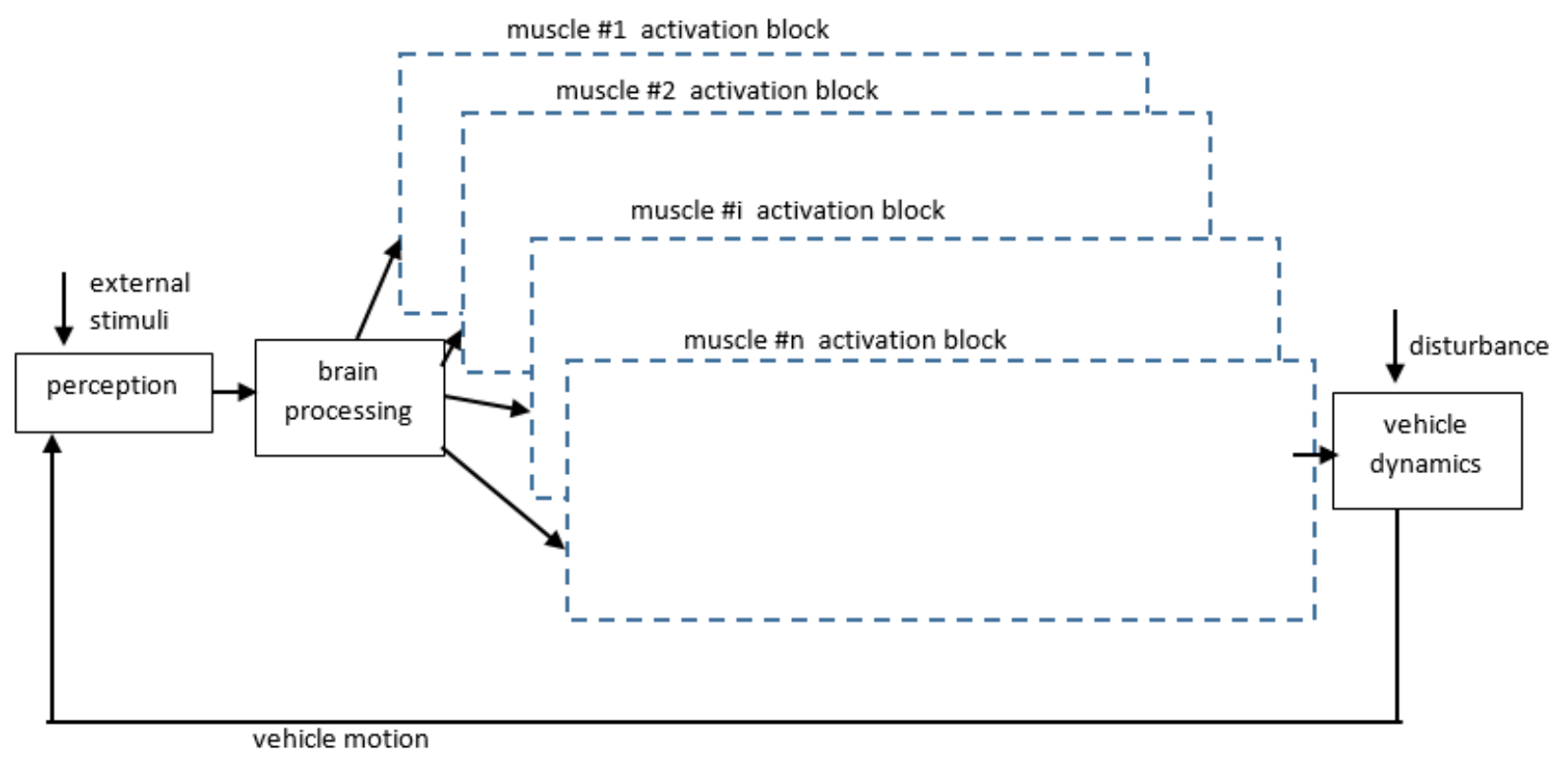

Fig. 2 - Evolution of the driver model in Fig. 1: possible scheme for driver-vehicle models of different complexity, taking into account the activation of the NMS of a number of muscles.

The ISW is able to capture the complexity of the activation of different muscles performing the driving task. This will probably shed some light on a realistic value of driver's reaction time.

\section{General Description and Concept Design of the ISW}

In Fig. 3 the ISW is presented. The ISW has six spokes and two handles. The ISW is made from Carbon Fibre Reinforced Polymer (CFRP) to keep the technical specifications listed in Tab. 1, precisely the mass properties of a common steering wheel. Two spokes carry both the handles and two load cells that sense, at each hand, three forces and three moments. More details on six-axis load cells are given in Section 4. More details on the ISW structure are given in Section 5. 


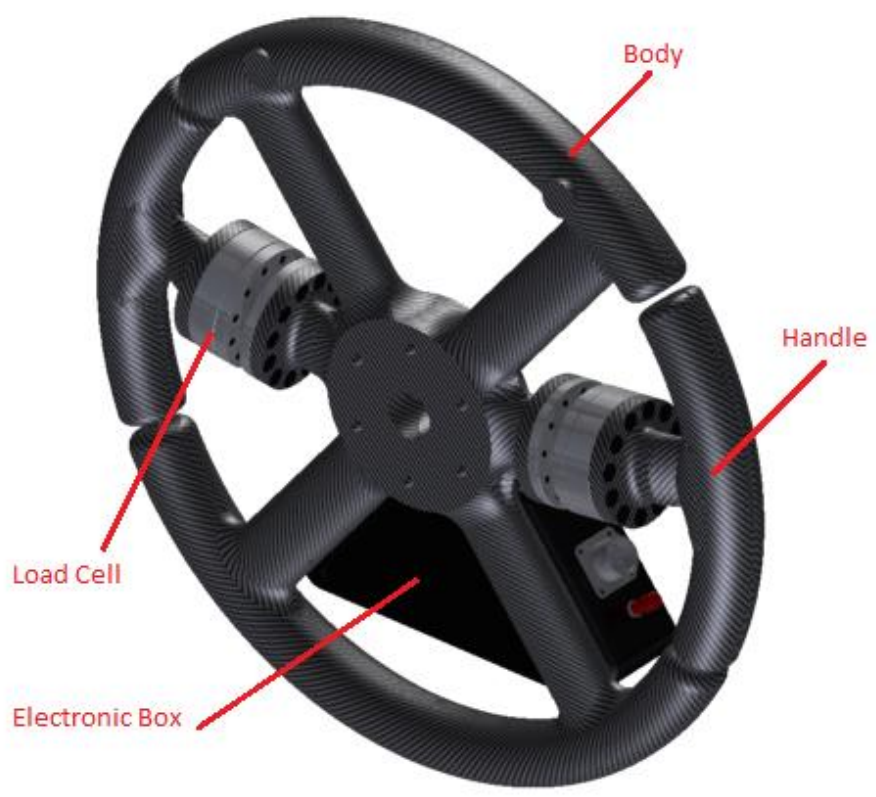

Fig. 3 - Instrumented Steering Wheel (ISW).

\begin{tabular}{lll}
\hline \hline \multicolumn{1}{c}{ Parameters } & \multicolumn{1}{c}{ Value } & \multicolumn{1}{c}{ Note } \\
\hline Mass & $2.7 \mathrm{~kg}$ & Common car SW \\
Diameter & $375 \mathrm{~mm}$ & Common car SW \\
Moment of inertia around rotation axis & $<0.030 \mathrm{~kg} \mathrm{~m}{ }^{2}$ & Common car SW \\
First natural frequency & $50 \mathrm{~Hz}$ & Common car SW \\
Rated Force Capacity & $\pm 250 \mathrm{~N}$ & ISW \\
Rated Moment Capacity & $\pm 25 \mathrm{Nm}$ & ISW \\
Maximum force (applied as pure load) & $5 \times$ Maximum sensed force & ISW \\
Maximum moment (applied as pure & $5 \times$ Maximum sensed & ISW \\
load) & moment &
\end{tabular}

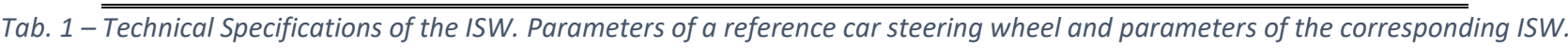

\section{Load Cells}

\subsection{Six-Axis Load Cells}

The six-axis load cell is based on an analytical model developed in [29].

The three spoke structure shown in Fig. 4 is connected with the outer frame by means of three patented 'sliding spherical joints' (they allow the sliding of the spoke tip along the axis of the spoke and three rotations around the spoke tip) [30]. By inspection of Fig. 4, two adjacent joints (1 and 3) act as a single virtual spherical joint $(1+3)$, so the structure is statically determined and it is possible to calculate the force and moments applied in the centre of the three spoke structure, if the forces acting at the tip of the spokes are known. 

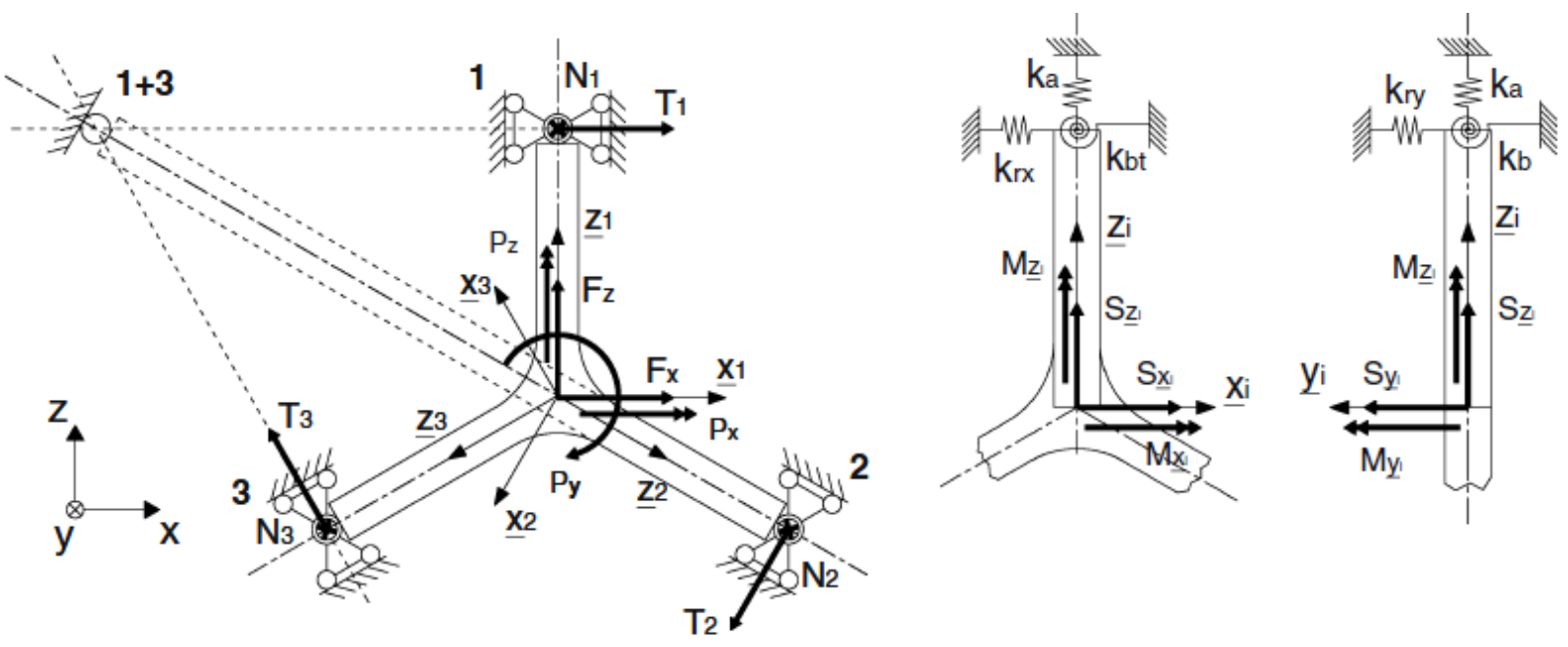

Fig. 4 -Model for the six-axis load cell, adapted from [30].

Each spoke is instrumented with four strain gauges located at the most stressed points and connected by means of half Wheatstone bridges (twelve strain gauges connected in six half bridges) as shown in Fig. 5 . The spokes are tapered to have a low gradient of strain at the zones in which the strain gauges are located.

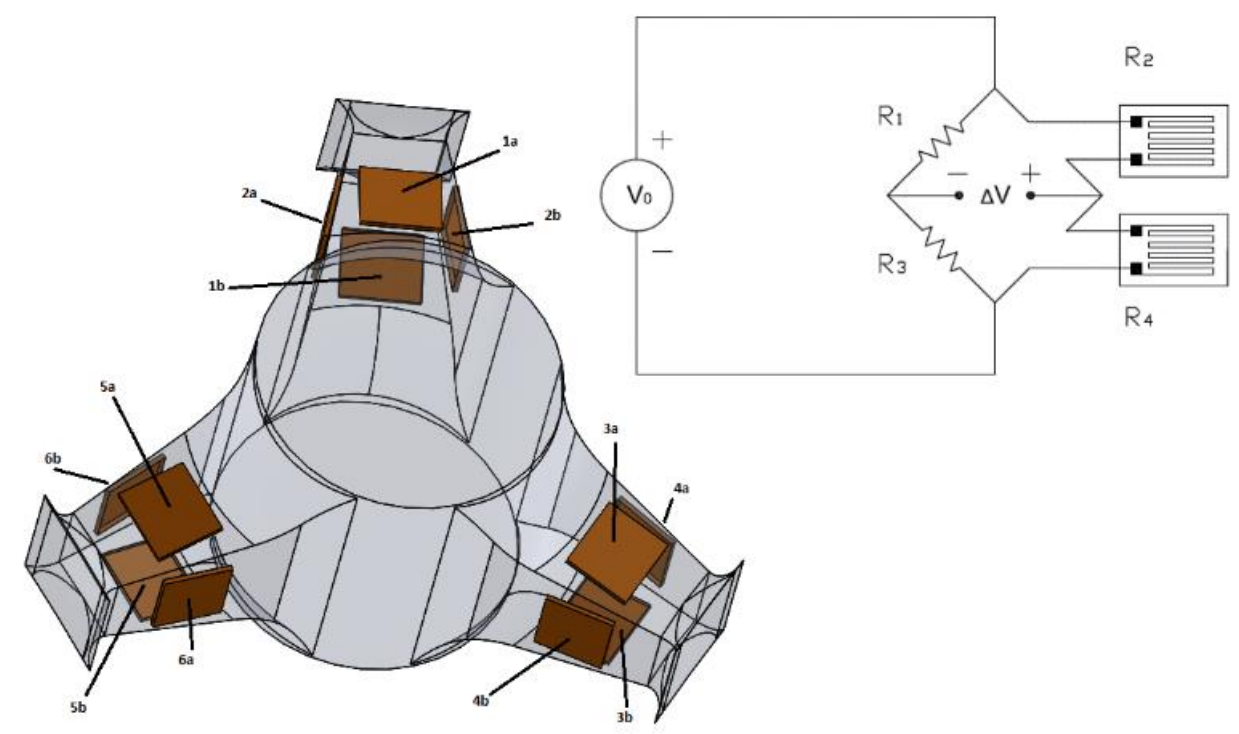

Fig. 5 - Location of the 12 strain gauges connected in half bridge configuration.

The three ideal joints are obtained by means of three laminae (Fig. 6 ), which introduce a non-zero stiffness in the spoke axial direction and non-infinite stiffness in the other two directions (lateral and tangential directions respectively). The three rotations at each spoke tip are associated to rotational springs. The springs acting at each spoke tip are shown in Fig. 4.

The output of the strain gauge is a voltage measurement, related to the deformation of the spoke due to the bending action (the connection in a half bridge allows to compensate the axial deformation). The deformations $\varepsilon_{i x}, \varepsilon_{i y}(i=1,3)$ at each of the three spokes read 


$$
\left\{\begin{array}{l}
\varepsilon_{i x}=\frac{1}{E W_{f x}}\left[M_{i x}+S_{i y}(L-h)\right] \\
\varepsilon_{i y}=\frac{1}{E W_{f y}}\left[M_{i y}-S_{i x}(L-h)\right]
\end{array}\right.
$$

where $h$ is the distance between the strain gauge and the tip of the spoke, $M_{i x}, M_{i y}$ and $S_{i x}, S_{i y}(\mathrm{i}=1,3)$ are respectively the moments and the forces acting at the spoke root, and $W_{f x, y}$ are the flexural moduli. In matrix form

$$
\underline{\varepsilon_{1}}=\left\{\begin{array}{l}
\varepsilon_{i x} \\
\varepsilon_{i y}
\end{array}\right\}=\frac{1}{E}\left[\begin{array}{cccccc}
0 & \frac{L-h}{W_{f x}} & 0 & \frac{1}{W_{f x}} & 0 & 0 \\
\frac{L-h}{W_{f y}} & 0 & 0 & 0 & -\frac{1}{W_{f y}} & 0
\end{array}\right]\left\{\begin{array}{c}
S_{i x} \\
S_{i z} \\
M_{i x} \\
M_{i y} \\
M_{i z}
\end{array}\right\}=\frac{1}{E}[\Phi] \underline{S_{i}}
$$

Combining the three strains at the three spokes

$$
\begin{gathered}
\left.\underline{E_{b}}=\left\{\begin{array}{l}
\varepsilon_{1} \\
\varepsilon_{2} \\
\varepsilon_{3}
\end{array}\right\}=\frac{1}{E}\left[\begin{array}{ccc}
{[\Phi]} & {[0]} & {[0]} \\
{[0]} & {[\Phi]} & {[0]} \\
{[0]} & {[0]} & {[\Phi]}
\end{array}\right]\left\{\underline{\frac{S_{1}}{S_{2}}}\right\} \underline{S_{3}}\right\}=\frac{1}{E}[\bar{\Phi}] \underline{S} \\
\underline{E_{b}}=\frac{1}{E}[\bar{\Phi}][\widehat{M}] \underline{F}
\end{gathered}
$$

Being $V_{0}$ the supply voltage of the strain gauges and $k$ the gauge factor, the voltage output $\Delta V$ is

$$
\Delta V=\frac{V_{0}}{4} k\left(\varepsilon_{1}-\varepsilon_{3}\right)=\frac{V_{0}}{4} k 2 \varepsilon_{1}
$$

Using the half-bridge configuration the two strain gauges measure the same strain $\left(\varepsilon_{1}=-\varepsilon_{3}\right)$. Substituting (3) in (4)

$$
\underline{\Delta V}=\frac{V_{0} k}{2 E}[\bar{\Phi}][\widehat{M}] \underline{F}=\left[C_{b}\right]^{-1} \underline{F}
$$

Where $C_{b}$ is the calibration matrix [6x6]. The use of half-bridge configuration allows the compensation of the thermal expansion effect.

\subsubsection{Design Optimization}

In the following, the optimization algorithm used to design the load cell is described. The first step for the optimization of the load cell is the definition of the loading conditions, the material parameters, the geometrical constraints and the design variables.

The design variables considered are related to the geometry of the spokes and the laminae i.e. length width, thickness of the lamina, fillet radius, width at the basis of the spoke in the two directions, width at the tip of the spoke in the two directions, position of the strain gauges (Fig. 5). A Sobol sequence (quasi-random) has been used [31]. 


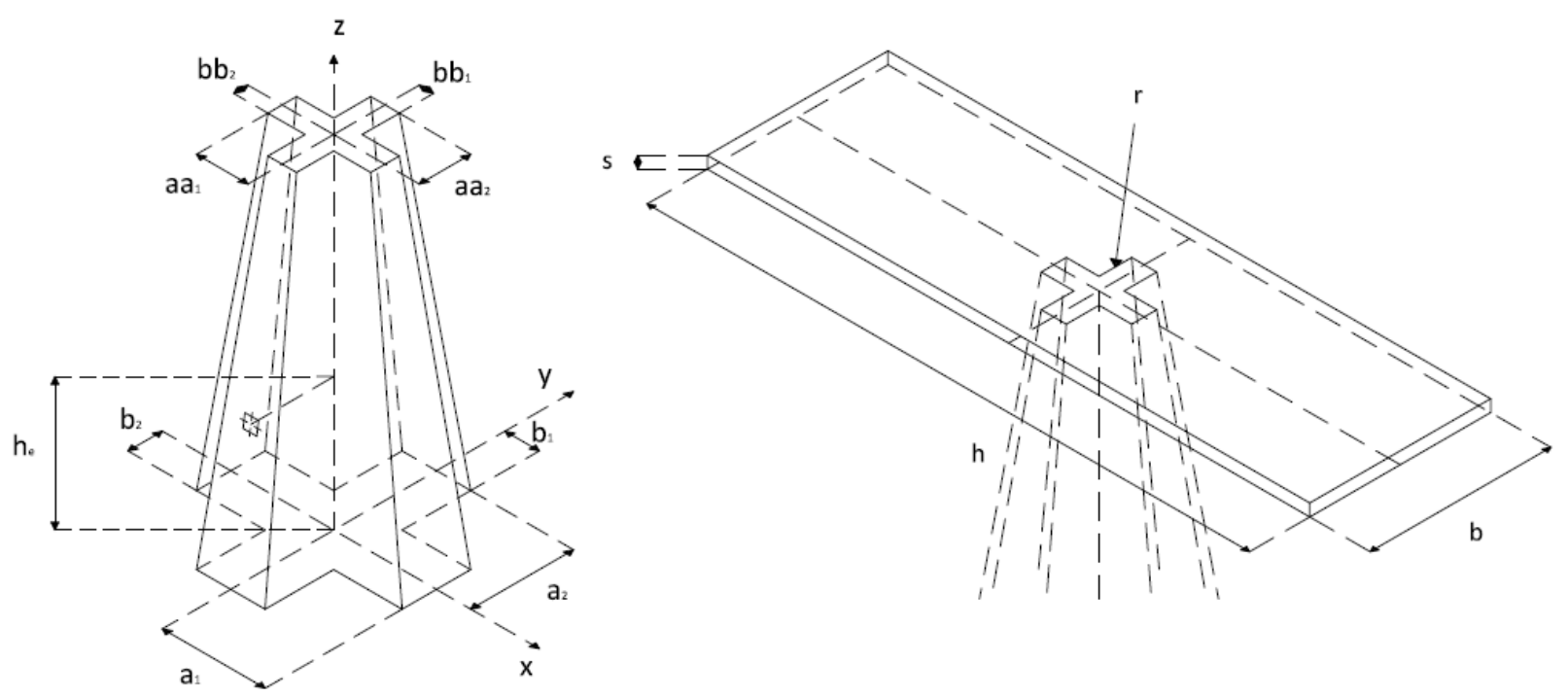

Fig. 5 - Design variables for the embodyment of the spoke and the lamina of the load cell..

The first constraint refers to the feasibility of the construction of the spokes and of the laminae. The second constraint refers to the maximum admissible stress of the material on both the spokes and the laminae. The stress is calculated with the formulae given in [32]. A number of loading cases are considered for designing the ISW. Finally, for every feasible solution, the calibration matrix $C_{b}$ is computed as described in Section 7. Four objective functions have been defined. The first objective function is related to the longitudinal force $F x$ sensitivity, the second to the lateral force Fy sensitivity, the third to the bending moment Mx sensitivity and the fourth to the torsional moment My sensitivity.

The multi-objective optimization design problem can be formulated as follows

$$
\begin{aligned}
& \min \quad f u n_{-} o b j\left(C_{b}(1,2), C_{b}(2,1), C_{b}(4,1), C_{b}(5,2)\right) \\
& a_{i}>b_{i} \\
& a a_{i}>b b_{i} \\
& a_{i}>a a_{i} \\
& b_{i}>b b_{i} \\
& \text { s.t. } \\
& l>2 a_{i} \\
& h_{e}<l+R \\
& \sigma<\sigma_{a d m} \\
& \text { geometry } \\
& a_{1}, b_{1}, a_{2}, b_{2}, a a_{1}, b b_{1}, a a_{2}, b b_{2}, h_{e}, h, s, b, r \in\left[\begin{array}{ll}
L B & U B
\end{array}\right]
\end{aligned}
$$

where the symbols refer to Fig.5. The Pareto optimal solutions are selected for the load cell design. Details on multi-objective optimization method are reported in [33].

\subsubsection{Finite Element Analysis}

After a preliminary design performed by the multi objective optimization method described in the previous section, a Finite Element Analysis is completed in order to get a more reliable configuration of the load cell. The CAD model has been imported in a FE code (Abaqus) as solid 3D deformable part and partitioned in the zones where the refinement of the mesh is needed. Due to the complex form of the load cell, tetrahedral quadratic elements (C3D10) were chosen for the mesh [34], in order to obtain a good compromise between the accuracy of the results and the computational time Fig. 6. The load cell material is Aluminium 7075-T6. 


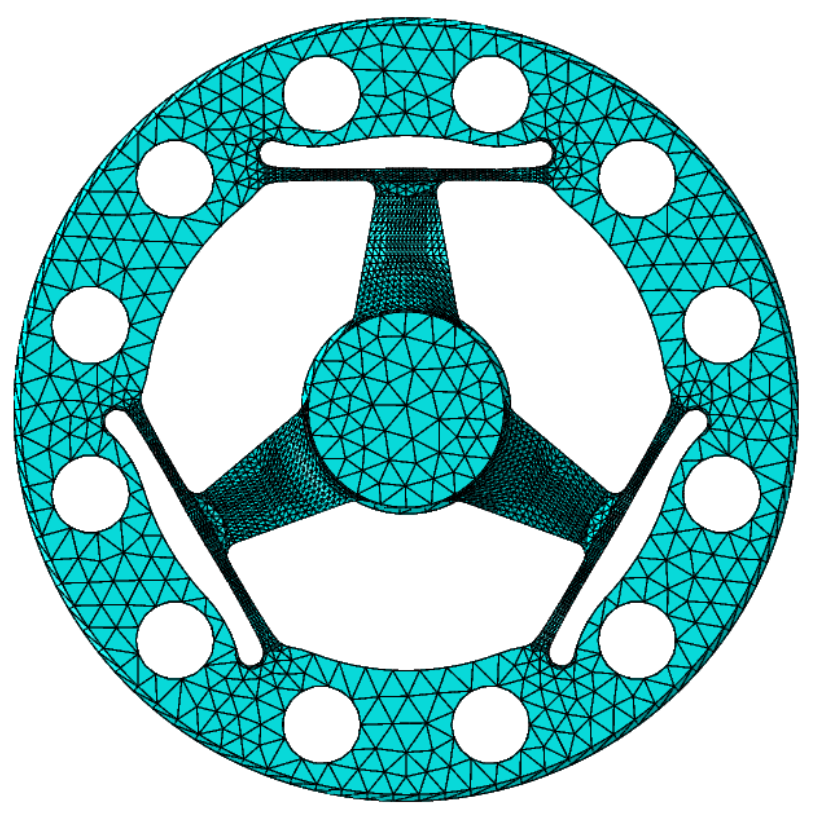

Fig. 6-FE Model of the load cell. Each spoke tip is linked via a lamina to the external ring.

The load cases considered in this analysis are the following (the reference system for forces and moments in Fig. 11)

1) Pushing the ISW with $\mathrm{Fx}=750 \mathrm{~N}$ (the moment is $\mathrm{Mz}=37.5 \mathrm{Nm}$ because the arm is $50 \mathrm{~mm}$ ); The maximum Von Mises stress is $280 \mathrm{MPa}$ which is less than the Tensile Yield Strength of the material (503 MPa).

2) Steering with $\mathrm{Fz}=750 \mathrm{~N}$ (the moment is $\mathrm{Mx}=37.5 \mathrm{Nm}$ because the arm is $50 \mathrm{~mm}$ ). The maximum Von Mises Stress is $256 \mathrm{MPa}$ which is less than the Tensile Yield Strength of the material (503 MPa).

3) Twisting the cell by applying $F x=500 \mathrm{~N}$ at the maximum distance from the cell axis ( $\mathrm{My}=50 \mathrm{Nm}$ and $\mathrm{Mz}=25 \mathrm{Nm}$ ). The maximum Von Mises Stress is $334 \mathrm{MPa}$ which is less than the Tensile Yield Strength of the material (503 MPa).

These three load cases are used to analyse the maximum stress status with the Von Mises equivalent stress in order to verify that is lower than the admissible stress for the material $\sigma_{V M}^{*}<\sigma_{a m m}$ where $\sigma_{a m m}$ is the yield stress for the material divided by a proper safety factor.

\subsection{Single Axis Load Cells}

Six single axis load cells (Omega LCM201 [35]) (three for each handle - Fig. 7) are used to measure the grip strength of the driver's hands. The grip strength $\left(F_{\text {grip }}\right)$ is given by Eq. 7, where $F_{x_{\text {six -axis load cell }}}$ is the force measured by the six-axis load cell in the axial direction and $F_{i}$ is the force measured by each of the handle load cells. The handle load cells measure only the force in the axial direction of the ISW.

$$
F_{\text {grip }}=\min \left(\left|F_{x_{\text {six-axis load cell }}}-\sum_{i=1}^{3} F_{i}\right|,\left|\sum_{i=1}^{3} F_{i}\right|\right)
$$

The three handle load cells are not aligned on the same axis to avoid the creation of a virtual hinge. 
All the voltage signals from the strain gauges of the six-axes load cells and from the six handle load cells, are received by the electronic board (Section 6) mounted in the electronic box of the ISW (see Fig. 3). The input power comes from the vehicle through the spring clock cable, and the signals are sent in digital form to the acquisition system through the same cable.

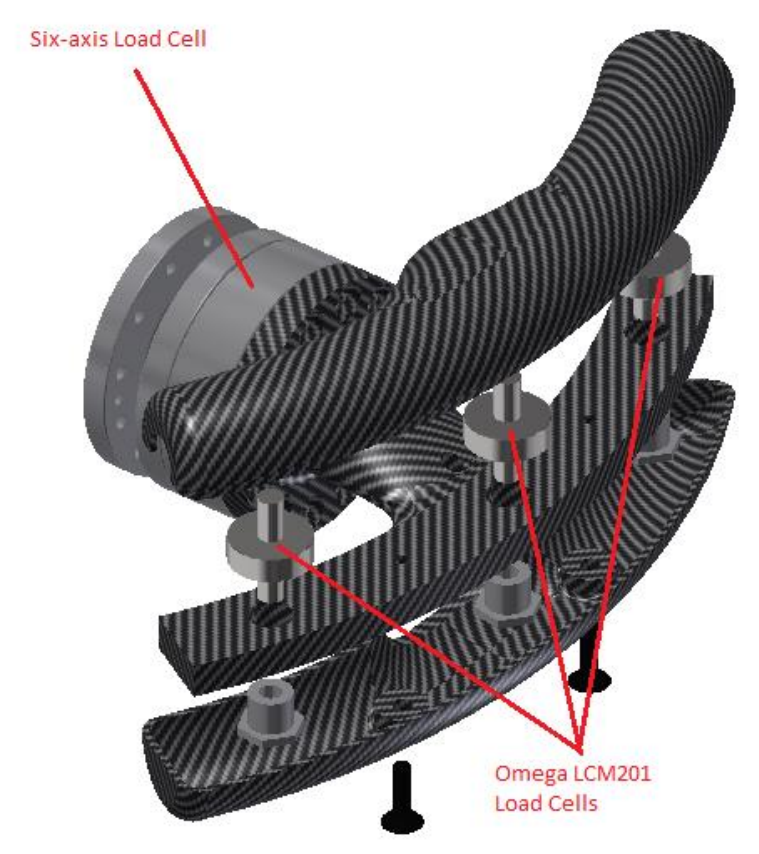

Fig. 7- Single axis load cells on the handle.

\section{ISW Modelling}

As discussed in Section 3, the ISW body is a carbon fibre laminate. Commercial pre-impregnated SAATI CC206 ET330S 45\% carbon fibre plies were used [36]. The ISW model for structural assessment is shown in Fig. 8. In the literature, many failure criteria for laminates [37] are introduced. The Tsai-Wu criterion [38] will be considered. Assuming that the lamina is orthotropic, in a plane stress state $\left(\sigma_{33}=0, \tau_{23}=0, \tau_{31}=0\right)$, the Tsai-Wu failure criterion requires that [34]

$$
F_{1} \sigma_{11}+F_{2} \sigma_{22}+F_{11} \sigma_{11}^{2}+F_{22} \sigma_{22}^{2}+F_{66} \tau_{12}^{2}+2 F_{12} \sigma_{11} \sigma_{22}<1
$$

$F_{1}, F_{2}, F_{11}, F_{22}, F_{66}, F_{12}$ are coefficients depending on experimental results on mono-directional fiberreinforced specimens under simple loading conditions and can be expressed as

$$
\begin{array}{llc}
F_{1}=\frac{1}{X_{t}}+\frac{1}{X_{c}} & F_{2}=\frac{1}{Y_{t}}+\frac{1}{Y_{c}} & F_{11}=-\frac{1}{X_{t} X_{c}} \\
F_{22}=-\frac{1}{Y_{t} Y_{c}} & F_{66}=\frac{1}{S_{12}^{2}} & F_{12}=f^{*} \sqrt{F_{11} F_{22}}
\end{array}
$$

$f^{*}$ is set to zero due the ignorance on the limit biaxial stress at failure. Under general biaxial loading, the TsaiWu failure criterion using $F_{12}=0$ predicts the composite failure with sufficient accuracy for practical applications [39].

The Tsai-Wu index is always lower than 1. The most critical area is the fillet between end of the spoke and handle. 


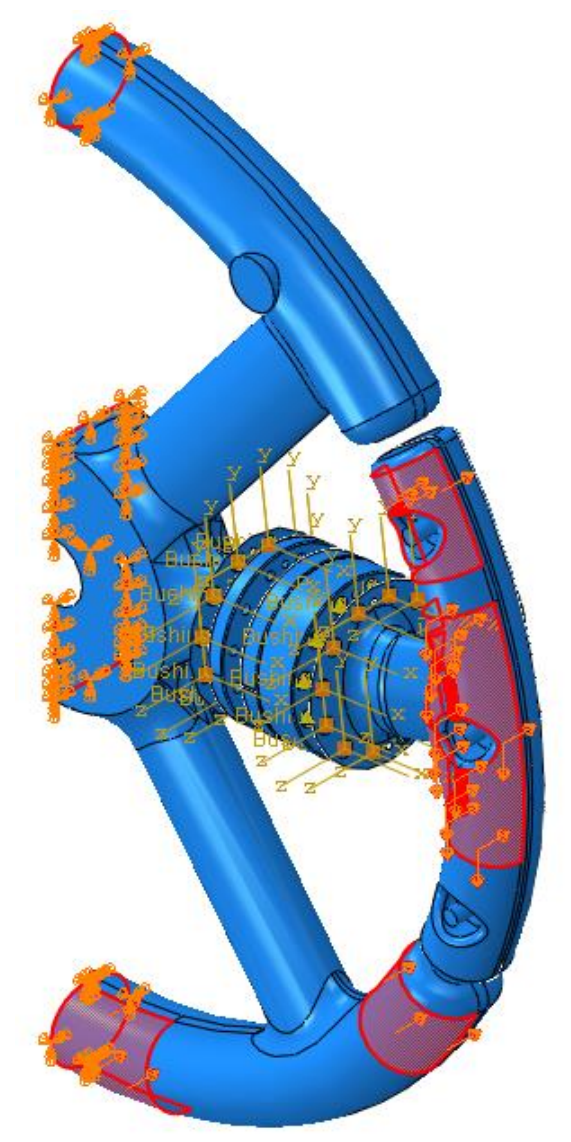

Fig. 8- Constraints and forces on the FE model.

A free-free dynamic analysis has been completed after the static one. Each mode was examined to avoid natural frequencies lower than $50 \mathrm{~Hz}$ (Tab. 1).

\section{Data Acquisition System Design}

A custom made signal processing electronic board, designed for the purpose and fitted in the electronic box of the ISW (Fig. 3), is able to supply power both to the strain gauge bridges of the two six-axes load cells and to the six uniaxial load cells for the grip strength measurement. The electronic board is powered by the vehicle electric system, and is able to process the output voltage signals of the load cells bridges. The electronic board output are the six forces and moments for each six-axes load cell and a single force for each of the uniaxial load cells.

Fig. 9 shows the flow chart of the signal processing for one six-axes load cell. The six half Wheatstone bridges output voltages are amplified with an amplifier gain G (100-1000). It is also possible to activate analog LP/HP filters. A digital potentiometer for each input channel is used for automatically zeroing the output of the Wheatstone bridge at system power up.

The 18 analog voltage signals are digitally converted through three synchronized 16 bits simultaneous sampling $A / D$ converters. The $A / D$ converters are connected in daisy-chain mode i.e. data from multiple devices are transmitted through the chain and the last device sends data to the SPI interface connected to the 16-bit digital signal processor (DSP). The signals are oversampled by a factor 8 and then decimated, to increase the resolution of 1.5 bits and the signal to noise ratio by a factor of 2.8 [40].

The DSP (dspic33) [41] uses the calibration matrix $C_{b}$ to obtain the forces $F$ (Eq. 16). Those output signals are sent through a CAN interface (ISO 11898-2 standard CAN2.0B, 1Mb/s, 5 frames with full 8 bytes payload including time stamp) or an USB cable to an acquisition system at a constant frequency of $750 \mathrm{~Hz}$ per channel. 


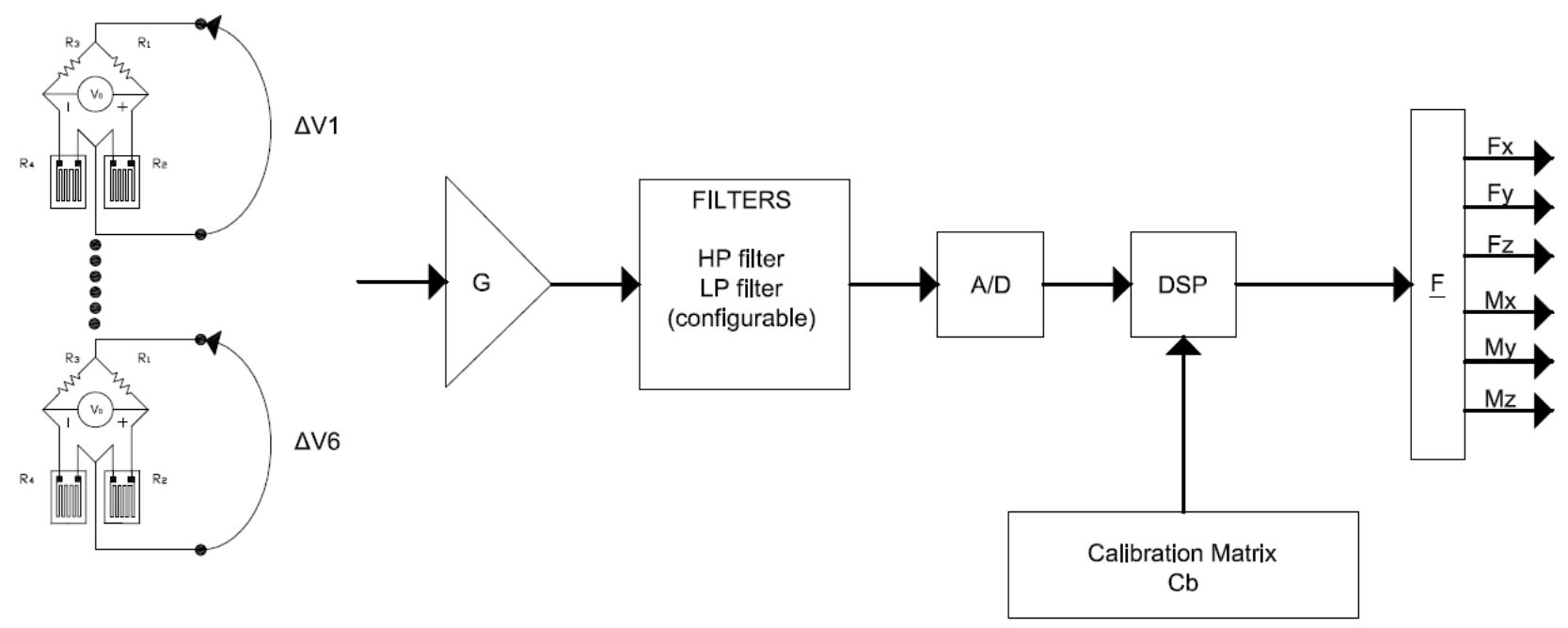

Fig. 9- Signal processing electronic board.

\section{Experimental Calibration}

\subsection{Static Calibration}

The calibration matrices $C_{b}$ (Eq. 16), loaded in the electronic board, are experimentally determined to accurately take into account the real behaviour of the components, the positioning error on the strain gauges, the analytical approximation, etc... For the experimental calibration process, a special fixture has been designed (Fig.10) to allow the alignment of the load cell to the axis of a test machine (MTS Synergie 200 [42]) in several angular and linear positions.

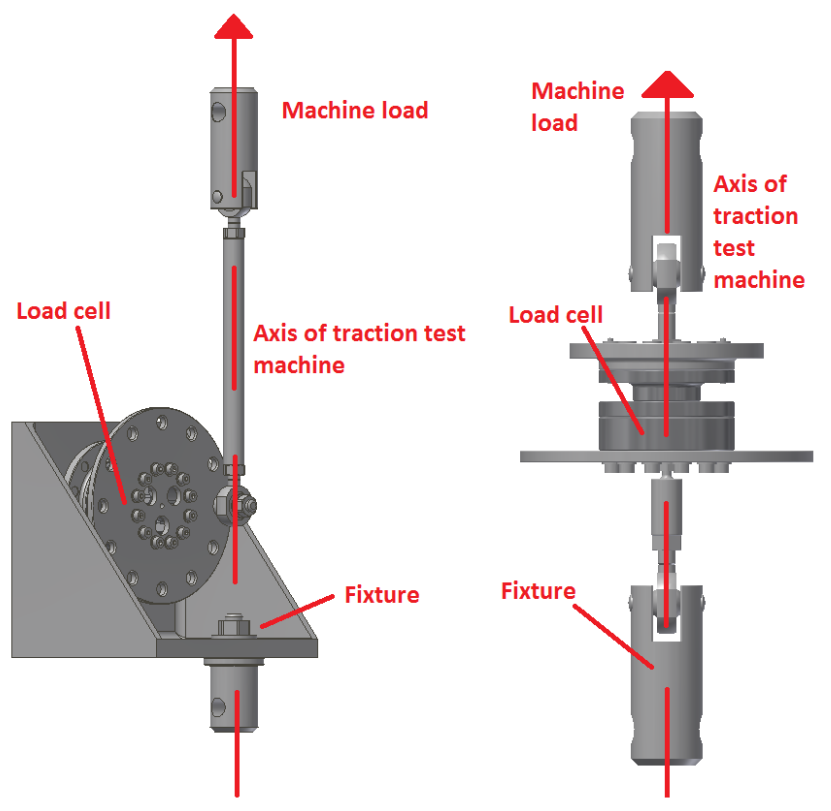

Fig. 10 -Calibration system.

This device allows more than 70 known load combinations, which could be related with the output signals from the strain gauges bridges using the least square method. Let be $\underline{F}^{(j)}$ the vector containing the forces and moments applied to the load cell by the traction test machine in the $j$-th experimental trial $(1 \leq j \leq n$, where $n$ is the total number of trials) 


$$
\underline{F}^{(j)}=\left[\begin{array}{c}
F_{x} \\
F_{y} \\
F_{z} \\
M_{x} \\
M_{y} \\
M_{z}
\end{array}\right]=\left[\begin{array}{c}
F_{1}^{(j)} \\
F_{2}^{(j)} \\
F_{3}^{(j)} \\
F_{4}^{(j)} \\
F_{5}^{(j)} \\
F_{6}^{(j)}
\end{array}\right]
$$

The $i$-th single term $F_{i}^{(j)}$ of the right vector is

$$
F_{i}^{(j)}=a_{i, 1} \Delta V_{1}^{(j)}+a_{i, 2} \Delta V_{2}^{(j)}+a_{i, 3} \Delta V_{3}^{(j)}+a_{i, 4} \Delta V_{4}^{(j)}+a_{i, 5} \Delta V_{5}^{(j)}+a_{i, 6} \Delta V_{6}^{(j)}=\left[\Delta V^{(j)}\right]^{T} \underline{a_{i}}
$$

Where $a_{i, 1}, a_{i, 2}, a_{i, 3}, a_{i, 4}, a_{i, 5}, a_{i, 6}$ are the term of the $i$-th row of the calibration matrix $C_{b}$ and $\Delta V_{1}^{(j)}, \Delta V_{2}^{(j)}$, $\Delta V_{3}^{(j)}, \Delta V_{4}^{(j)}, \Delta V_{5}^{(j)}, \Delta V_{6}^{(j)}$ are the strain gauge bridge output of the $j$-th trial.

Exploiting the equations for all the six load components it is possible to obtain, in matrix form

$$
\begin{gathered}
{\left[\underline{F_{1}}, \underline{F_{2}}, \underline{F_{3}}, \underline{F_{4}}, \underline{F_{5}}, \underline{F_{6}}\right]=\left[\begin{array}{c}
\Delta V^{(1)} \\
\vdots \\
\Delta V^{(j)} \\
\vdots \\
\Delta V^{(n)}
\end{array}\right]^{T}\left[\underline{a_{1}}, \underline{a_{2}}, \underline{a_{3}}, \underline{a_{4}}, \underline{a_{5}}, \underline{a_{6}}\right]} \\
{[F]=[\Delta \mathrm{V}]^{T}\left[C_{b}\right]^{T}}
\end{gathered}
$$

Let $[e]^{T}$ be the error between the force and moment measured by the six-axes load cell $[F]^{T}$ and the known force and moment applied by the test machine $[(\widetilde{F})]^{T}$

$$
[e]^{T}=[F]^{T}-[\tilde{F}]^{T}=[\Delta V]^{T}\left[C_{b}\right]^{T}-[\tilde{F}]^{T}
$$

The squared error $e^{2}$ can be expressed as

$$
e^{2}=\left\|[e]^{T}\right\|_{F}^{2}=\operatorname{tr}\left([e][e]^{T}\right)=\operatorname{tr}\left(\left[C_{b}\right][\Delta V][\Delta V]^{T}\left[C_{b}\right]^{T}+[\tilde{F}][\tilde{F}]^{T}-\left[C_{b}\right][\Delta V][\tilde{F}]^{T}-[\tilde{F}][\Delta V]^{T}\left[C_{b}\right]^{T}\right)
$$

Where $\|\cdot\|_{F}$ is the Frobenius matrix norm and $\operatorname{tr}(\cdot)$ is the matrix trace [43]. The least square method consists in finding the $\left[C_{b}\right]$ matrix terms, to solve the squared error minimization problem.

$$
\frac{\partial e^{2}}{\partial\left[C_{b}\right]^{T}}=2[\Delta V][\Delta V]^{T}\left[C_{b}\right]^{T}-2[\Delta V][\tilde{F}]^{T}=0
$$

From Eq. (15) it is possible to obtain the calibration matrix $\left[C_{b}\right]$ that solves the error minimization problem.

$$
\left[C_{b}\right]=[\tilde{F}][\Delta V]^{T}\left([\Delta V][\Delta V]^{T}\right)^{-1}
$$

The minimization problem is well posed if the number of independent trials $n$ is grater than the rank of the matrix $[\Delta V]$. In order to define the calibration matrix in a robust way, the minimization algorithm should exploit an adequate number of independent trials.

The experimental calibration matrix obtained has the first three rows expressed in $[N / V]$ and the last three rows in $[\mathrm{Nm} / \mathrm{V}]$. Each row of the calibration matrix is related with a load component. It is possible to notice that some matrix terms are very low with respect to others, this means that the contribution of some bridges 
on certain forces and moments is not relevant. Theoretically the contribution of those bridges should be null, but the non-ideal behaviour of the spherical joints, the errors in the strain gauges positioning, the machining errors made the contribution of those bridges not vanishing.

$$
\left[C_{b, \exp }\right]=10^{4} \cdot\left[\begin{array}{cccccc}
-0.167 & -24.266 & -1.067 & 12.315 & -0.106 & 12.127 \\
19.208 & -0.439 & 19.191 & 0.604 & 19.647 & 0.422 \\
-0.210 & 0.133 & -1.855 & 21.080 & 0.583 & -21.459 \\
-0.3715 & 0.0146 & 0.1841 & -0.0041 & 0.1826 & 0.0074 \\
-0.0007 & -0.3587 & 0.0326 & -0.3553 & 0.0115 & -0.3585 \\
-0.0015 & -0.0118 & 0.3047 & 0.0089 & -0.3193 & 0.0041
\end{array}\right]
$$

It is possible to compare the terms of the experimental calibration matrix with the same terms of the matrix obtained by the FE model presented in Section 4.

$$
\left[C_{b, F E M}\right]=10^{4} \cdot\left[\begin{array}{cccccc}
0.0000 & -25.3318 & 0.0765 & 12.6658 & -0.0765 & 12.6668 \\
21.7835 & 0.0000 & 21.7830 & 0.0000 & 21.7835 & 0.0003 \\
0.0883 & 0.0003 & -0.0440 & 21.9388 & -0.0443 & -21.9400 \\
-0.4135 & 0.0000 & 0.2068 & 0.0025 & 0.2068 & 0.0003 \\
0.0000 & -0.4078 & 0.0000 & -0.4078 & 0.0000 & -0.4078 \\
0.0000 & 0.0003 & 0.3583 & 0.0000 & -0.3583 & 0.0000
\end{array}\right]
$$

The two matrices are in a very good agreement.

\subsection{Static and Dynamic Force Compensation}

The load cells measure the static weight of the handles and of the mono axial load cells used for the grip measurement. These static contributions act on the measured forces depending on the ISW angular position, and they must be compensated. Knowing the sum of the masses of each component attached to the load cell $m_{H}$, the steering angle $\delta$ corrected by the vehicle roll, the ISW inclination $\beta$ with respect to the vertical axis corrected by the vehicle pitch (see Fig. 11), it is possible to calculate the static weight compensation $\vec{F}_{W}$ as follows

$$
\vec{F}_{W}=\Lambda_{\text {weight }}(\delta, \beta) \cdot m_{H} \cdot g
$$

Moreover, the load cells can rotate around the steering axis, so the measured forces $\vec{F}_{\text {meas }}$ are in a noninertial reference frame and inertial forces $\vec{F}_{\text {inertial }}$ act on the cells themselves. Such inertial forces must be compensated to measure the correct forces applied by hands $\vec{F}_{\text {hand }}$ on the ISW.

$$
\vec{F}_{\text {meas }}=\vec{F}_{\text {hand }}+\vec{F}_{\text {inertial }}=\vec{F}_{\text {hand }}-m_{H} \vec{a}_{v}-m_{H} \frac{d \vec{\Omega}}{d t} \times \vec{r}_{O H}-m_{H} \vec{\Omega} \times\left(\vec{\Omega} \times \vec{r}_{O H}\right)-2 m_{H} \vec{\Omega} \times \vec{v}_{H}
$$

where $\vec{a}_{v}$ is the vehicle acceleration with respect to the inertial reference frame, $\vec{\Omega}$ is the rotational speed of the handle taking into account the steering velocity, the roll and the pitch motion, $\vec{r}_{O H}$ is the distance of the handle CoG (Centre of Gravity) from the centre of the inertial reference frame and $\vec{v}_{H}$ is the speed between the handle and the inertial reference frame. The contribution of the inertial forces on the load cell is shown in Fig. 12. It is clear that compensation is needed to obtain a reasonable instrument resolution, i.e. without compensation, the accuracy of the ISW would not be acceptable. 

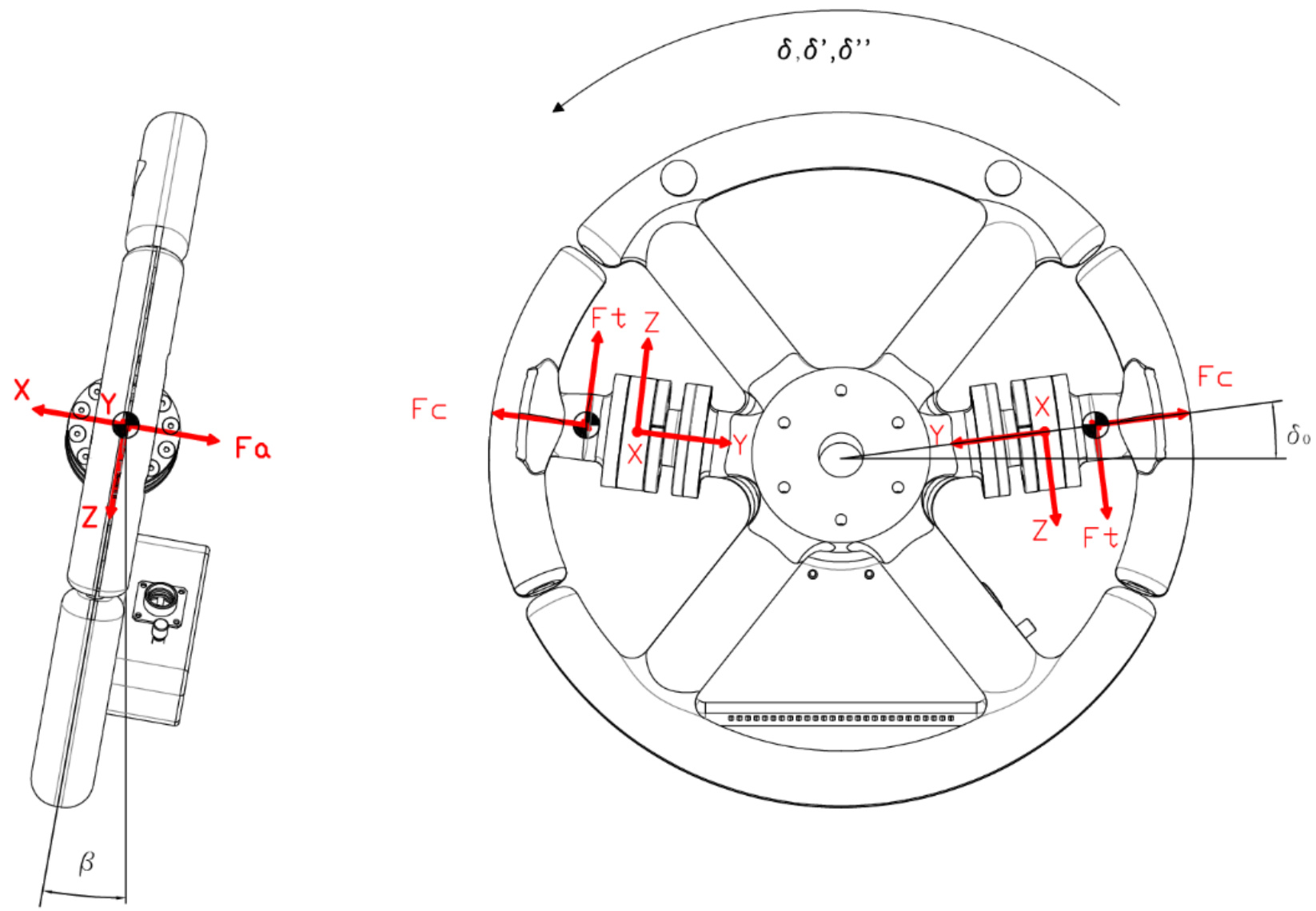

Fig. 11 - Reference frames for both load cells and inertial forces.

Right Hand - Force Compensation

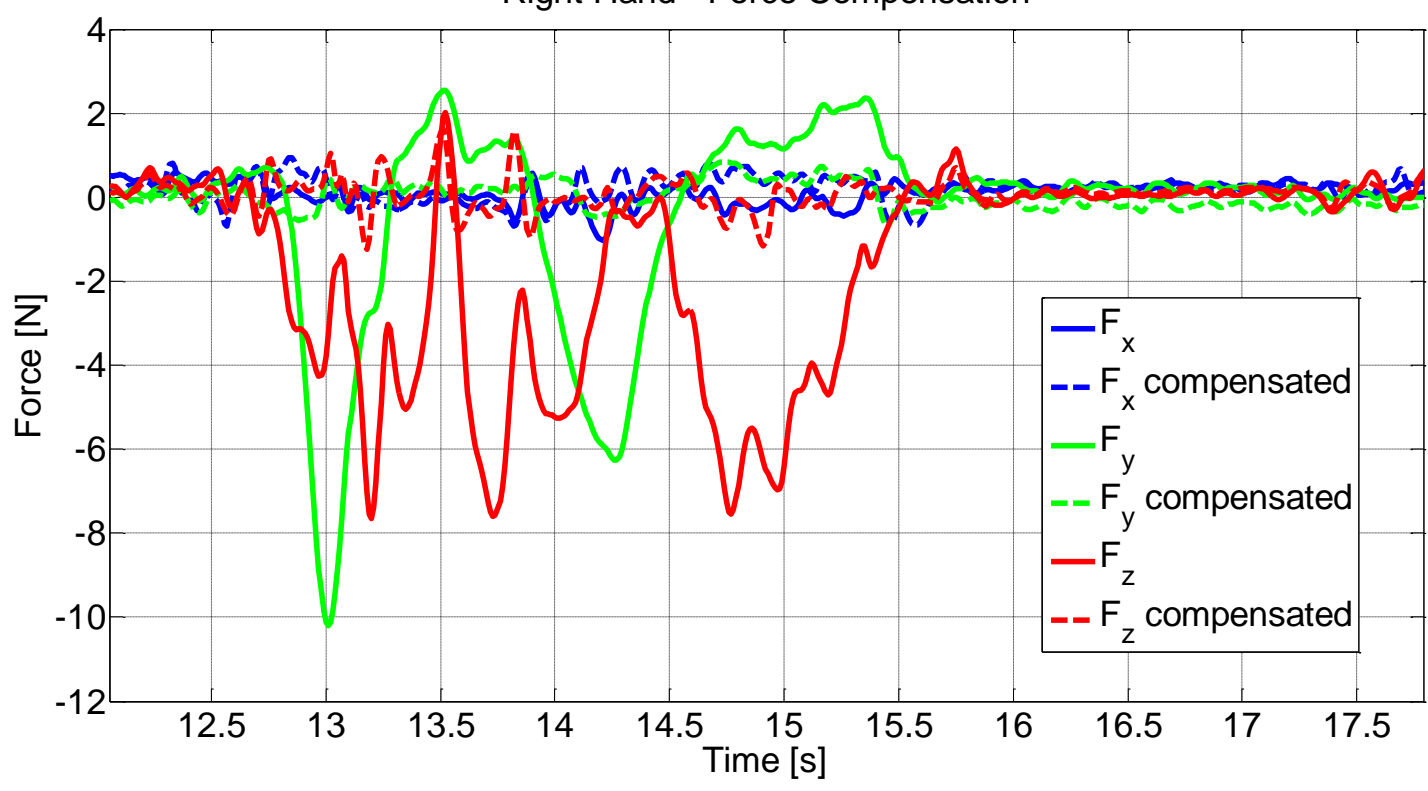

Fig. 12 - Weight and inertial forces compensation on the right load cell during a driving manoeuvre. Compensating $F_{z}$ and $F_{y}$ is extremely important (forces and moments are shown in Fig.11)

\section{Error Analysis and Measuring Performance}

Referring to error analysis, in Fig. 13 the distribution of the residuals is shown. Plotting on a graph the measured forces and moments versus the applied forces and moments, it is possible to check visually the [Digitare qui] 
linearity of the load cell (see Fig. 14). The linearityof the ISW sensor is given in Tab.2, according to UNI EN ISO 376.
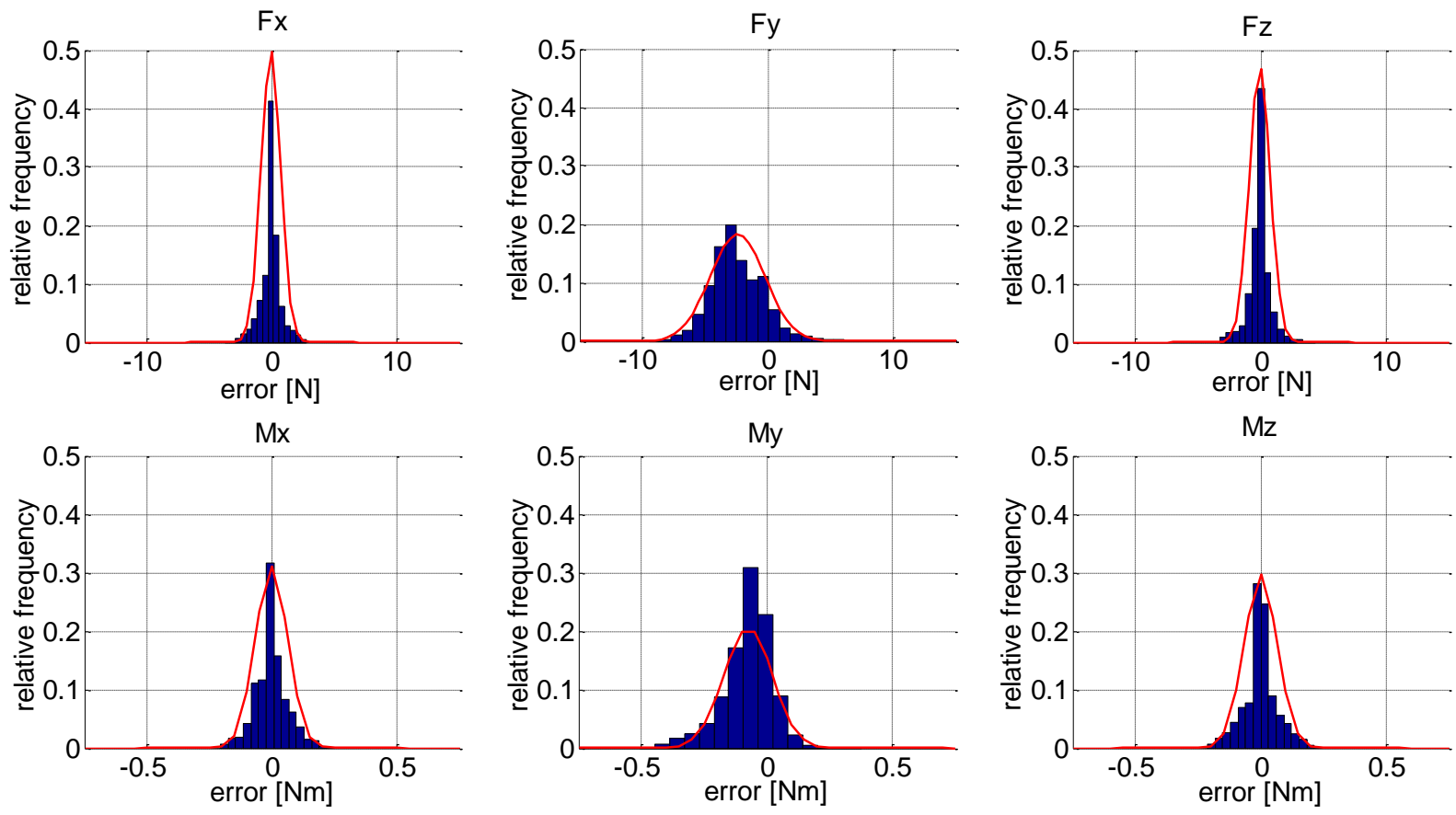

Fx
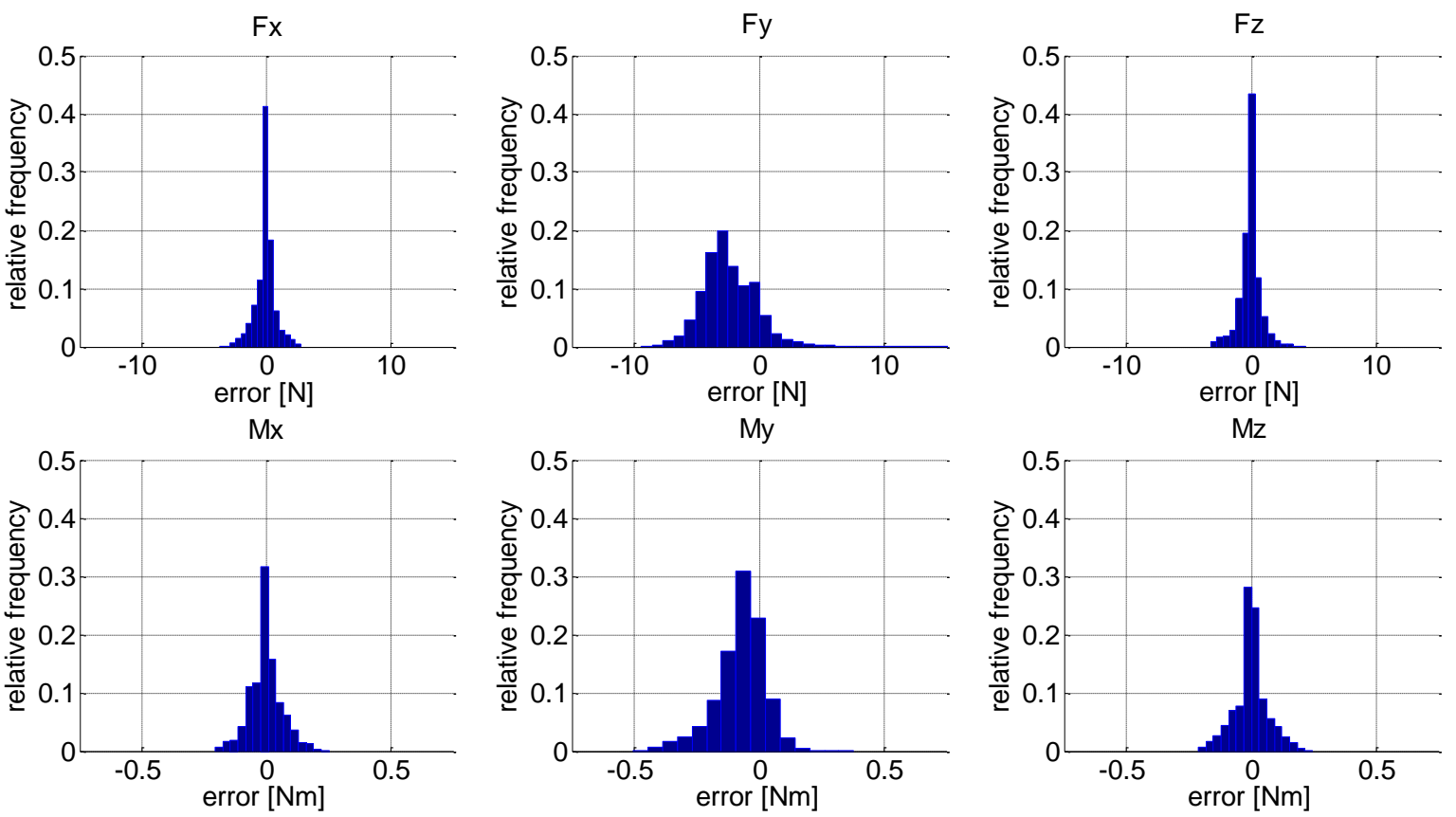

Fig. 13 -Distribution of the residuals in calibration. 

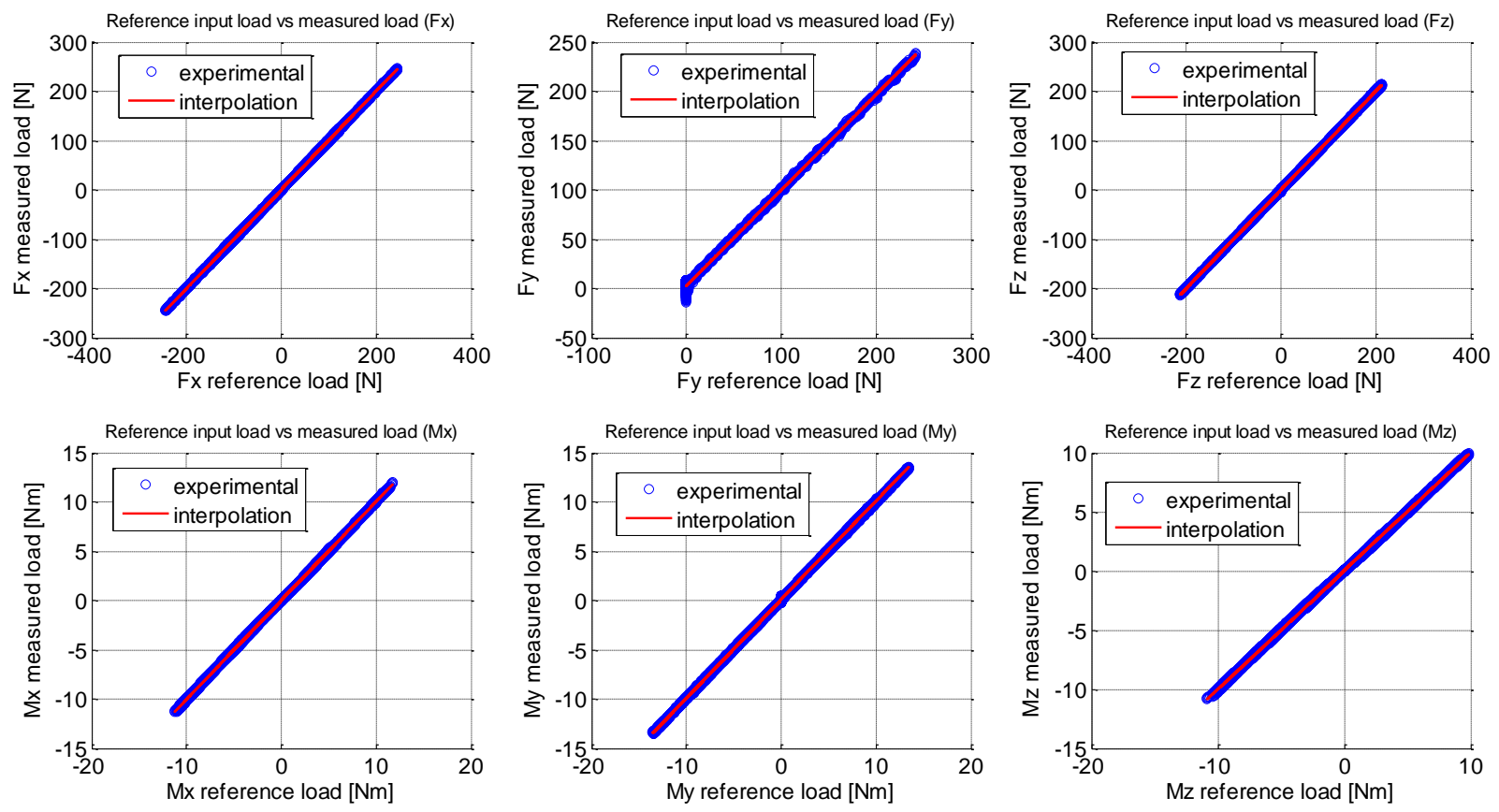

Fig. 14 - Load cell linearity.

By inspection of Tab.2, we see that for each force and moment component, a unitary value of the determination coefficient $R^{2}$ means a good approximation of the experimental data with the linear model.

\begin{tabular}{ccc}
\hline \hline Load & $e_{\text {lin }}[\%$ Full Scale $]$ & $R^{2}$ \\
\hline$F_{x}$ & 0.154 & 0.99996 \\
$F_{y}$ & 0.244 & 0.99660 \\
$F_{z}$ & 0.167 & 0.99995 \\
$M_{x}$ & 0.125 & 0.99990 \\
$M_{y}$ & 0.094 & 0.99997 \\
$M_{z}$ & 0.126 & 0.99990
\end{tabular}

$\overline{\text { Tab. } 2 \text { - Linearity errors during calibration of a load cell. }}$.

The ISW measurement performance is given in Tab.3 (the FS for the Accuracy and Linearity error calculation are intended the same as the rated capacity of the sensor).

\begin{tabular}{ccccc}
\hline \hline Load & $\begin{array}{c}\text { Rated Capacity } \\
{[\mathrm{N}]-[\mathrm{Nm}]}\end{array}$ & $\begin{array}{c}\text { Resolution } \\
{[\mathrm{N}]-[\mathrm{Nm}]}\end{array}$ & $\begin{array}{c}\text { Accuracy } \\
{[\% \mathrm{FS}]}\end{array}$ & $\begin{array}{c}\text { Linearity Error } \\
{[\% \mathrm{FS}]}\end{array}$ \\
\hline$F_{x}$ & 250 & 0.01 & \pm 0.29 & 0.15 \\
$F_{y}$ & 250 & 0.01 & \pm 0.51 & 0.24
\end{tabular}




\begin{tabular}{ccccc}
$F_{z}$ & 250 & 0.01 & \pm 0.31 & 0.16 \\
$M_{x}$ & 25 & 0.001 & \pm 0.26 & 0.12 \\
$M_{y}$ & 25 & 0.001 & \pm 0.18 & 0.09 \\
$M_{z}$ & 25 & 0.001 & \pm 0.26 & 0.12 \\
\hline \hline
\end{tabular}

Tab. 3 - Measurement performance of the ISW.

\section{Experimental Frequency Analysis}

An experimental modal analysis of the ISW is performed to check the dynamic performance. Tab. 4 shows the positioning of accelerometers. All of the measured frequencies are fully acceptable since they are considerably higher than the natural frequency of the reference/current mass production steering wheel, equal to $50 \mathrm{~Hz}$ (Tab. 1). The FE model predicts quite accurately the natural frequencies of the ISW.

\begin{tabular}{|c|c|c|}
\hline Position & Frequency & Frequency \\
\hline & Measured: \\
1 st $: 243-260 \mathrm{~Hz}$ \\
$2 \mathrm{nd}: 287-296 \mathrm{~Hz}$
\end{tabular}

Tab. 4 - Natural frequency analysis.

\section{Testing the ISW on a vehicle}

The ISW has been fitted on a Toyota Auris [45], Fig. 15. The standard production spring clock cable provided power to the ISW and signals connection. The ISW data were acquired via a Kvaser USBcan Light system [46] and logged into a laptop with the steering angle data provided by the vehicle embedded sensor via CAN and the data provided by a OXTS RT3000 inertial and GPS system [47]. 

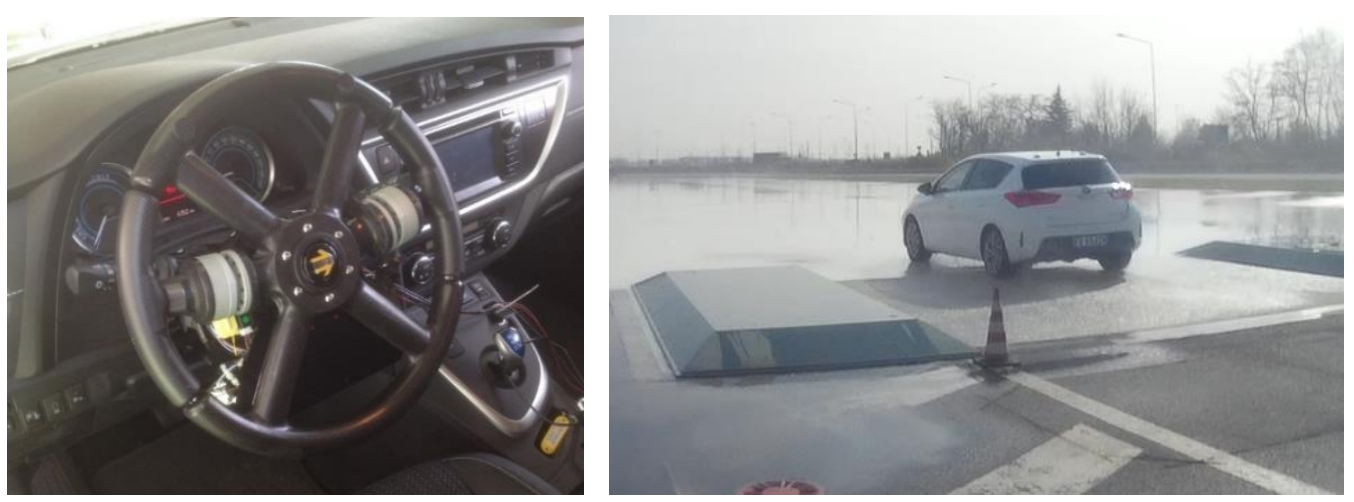

Fig. 15 -Left: Instrumented Steering Wheel fitted on a Toyota Auris car. Right: Kick-plate at Pista e Centro Guida Sicura ACI-Sara Lainate, near Milan, Italy.

The tests were performed at the track "Pista e Centro Guida Sicura ACl-Sara Lainate" [48] (Fig. 15). The track is equipped with a kick-plate. The kick-plate is basically a plank that is suddenly displaced in lateral direction when the rear axle of a vehicle passes on it. This lateral excitation causes a yaw motion of the vehicle. Since the road surface that the vehicle traverses after the kick-plate is wet, the yawing motion amplitudes are relevant. To keep a straight path, the driver is required to counteract the yaw produced by the kick-plate. If the action of the driver is not quick, a spin may occur. The strong driver's action that is required implies quite different forces and moments exerted by the two hands on the ISW.

Ten different drivers performed the kick-plate test nine times each, with two different speeds and two different kick gains (three times low speed - low gain, three times high speed - low gain, three times low speed - high gain). The kick direction was random during all the tests.

Fig. 16 refers to a kick-plate manoeuvre. Two time-histories are shown, one for the left hand and one for the right hand (see Fig. 11 for the reference systems). It is possible to note that the driver applies forces of less than $50 \mathrm{~N}$ during the emergency maneuver. The moments applied by the two hands are quite different from each-other and are lower than $5 \mathrm{Nm}$. The grip forces at each handle are approximately in phase with the applied $F_{x}$ and $F_{y}$.


Fig. 16- Kick-plate manoeuvre (input towards the left). Forces and moments measured by the Instrumented Steering Wheel at the two hands. LCi refers to the $i$-th single axis load cell for grip detection.

[Digitare qui] 

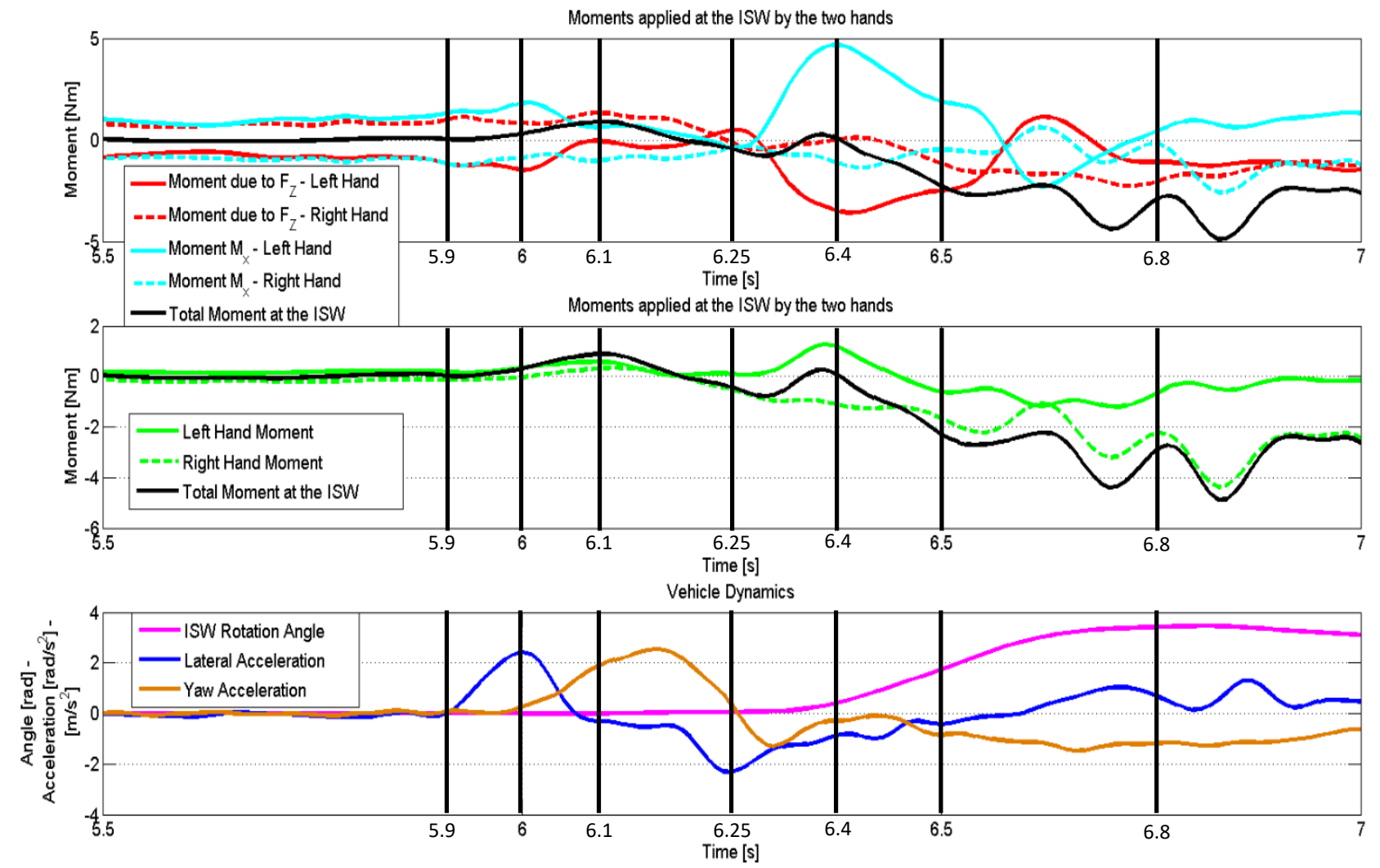

Fig. 17- Kick-plate manoeuvre (input towards the left). Moments due to tangential forces and bare moments applied by hands during the initial phase of steering.

The analysis of Fig.17 may provide hints to develop a new driver model. A kick-plate manoeuvre towards the left is considered. The first instants of the manoeuvre are considered. Before $t=5.9 \mathrm{~s}$ the moment at the ISW produced by the force at one hand is almost opposed by the bare moment produced by the same hand. A kind of a moment preload on the steering wheel is produced by the two hands, i.e. the two hands almost counteract each other in applying their moments on the ISW. This means that several NMS are activated in a coordinated manner. At $t=5.9 \mathrm{~s}$ the kick-plate starts its action. At $\mathrm{t}=6 \mathrm{~s}$ the maximum lateral acceleration of the vehicle is reached. The ISW rotation angle is still zero. At $t=6.1 \mathrm{~s}$ a non negligible moment is applied to the ISW, but the ISW rotation angle is still zero. Such non negligible moment seems counteracted by friction in the steering system, deliberately introduced to filter disturbances. The NMS is clearly activated in reflex mode, due to the lateral kick that the driver's body and arms have received. The total non negligible reflex moment is actually given by the two arms, wrists and hands, separately. At 6.25 s the voluntary steering action seems initiating, but he ISW rotation angle is still zero. At $6.4 \mathrm{~s}$ the maximum bare moment by the left hand is applied, partially counteracted by the moment due to the tangential force $F z$ at the same left hand. The ISW starts rotating. At $6.8 \mathrm{~s}$ the rotation angle is fully developed. About $1 \mathrm{~s}$ is elapsed since the kick-plate has applied the lateral acceleration.

After the analysis made above, we can suggest that a driver model should consider the activation of a number of Neuro-Muscular Systems, including reflex. We argue that a driver model with such features could capture accurately the actual moment applied to the steering wheel.

\section{Discussion}

We have tested the Instrumented Steering Wheel during a recovery/emergency manoeuvre, after a spin was initiated by passing with the car over a kick-plate. We have been able to record that the total moment applied 
at the steering wheel depends not only on forces applied by hands but also on moments. The Neuro-Muscular Systems of the two arms seem activated independently from each other. We could identify, considering an arm, the activation of different Neuro-Muscular Systems for the arm subsections, e.g. the activation of three spatial rotations of the wrists, the activation of the grip, and so on. Is this step important for scientific research? The discussion is open. It is evident that having an accurate tool like the ISW can be helpful to identify driving patterns and thus having information on how humans activate the steering system of vehicles. Obviously covering this gap is out of the scope of the paper. More analyses will have to be undertaken to monitor simple straight driving or curving manoeuvres, like lane change or evasion. In these cases, the interaction of the driver with the steering wheel is surely different with respect to the kick-plate manoeuvre we studied. In fact, we may imagine that the reflex loop is more activated in the kick-plate manoeuvre rather than during a lane change or an evasive manoeuvre. Actually in the first case, the vestibular system is stimulated, in the second case the visual system is more active. This occurrence does not affect the scope of the paper. Actually, in the paper, a new tool is described for future studies on the interaction between the driver and the steering wheel.

Crucial aspects in deriving driver models refer to behavioural and psychological issues. According with [5], we could say that automotive engineers do think of driver models as robots, i.e. machines that in a more or less deterministic way respond to external inputs. This is obviously not the case with humans, actually, behavioural and psychological issues may affect driving manoeuvres. At the moment the most advanced driver models are hardly including behavioural and psychological aspects [1,5,6-10]. We leave the implementation into driver models of such behavioural and psychological aspects to forthcoming studies. As a first step to improve driver models we envisage the proper modeling of the activation of different NMS pertaining to arms and their parts.

\section{Conclusions}

In this paper, an Instrumented Steering Wheel (ISW) able to measure the forces and moments applied by each hand of the driver during actual manoeuvres has been designed, manufactured, calibrated and used. The aim was to provide to the scientific community an accurate tool enabling the derivation of new driver models in which the activation of the Neuro-Muscular System could be included. Such envisaged models could provide an enhancement with respect to the current available driver models that do not appear accurate enough for developing effective controls for ADAS or AV.

The ISW has been modelled via original analytical and numerical design tools. The core part of the ISW, namely the two six axis load cells, have been optimally designed. The structural strength and the vibration modes of the ISW have been computed in advance and appeared to be close to the experimental measurement data. Such data match with the technical specifications which require the ISW to show mechanical features (inertia properties and eigenfrequencies) similar to the ones of current steering wheels. The high accuracy of the ISW has been obtained by compensating the spurious forces and moments acting on the handles due to gravity, inertia and vehicle motion. A specific analog/digital data acquisition system has been designed and tested. The electronic board is capable of processing the analog signals produced by twelve strain gauges and six mono-axial load cells. The output data are forces and moments and grips forces at the two handles (obtained by multiplying the raw signals by experimentally derived calibration matrixes). The ISW measuring performance has been evaluated in terms of accuracy and linearity, finding an accuracy ( $\pm 2 \sigma \%$ full scale) of $0.5 \%$ FS for forces and $0.3 \%$ FS for the moments. The technical specifications of the ISW seem unique, according to the Authors knowledge.

The ISW has proved its capability to suggest information for the subsequent derivation of new driver models in which the activation of the Neuro-Muscular System can be considered. The ISW has been used in a test track by a panel of drivers engaged in a series of recovery manoeuvres. The preliminary outcomes of the [Digitare qui] 
analysis of measured forces and data provided by the ISW show that the two hands and wrists and arms of the driver do act in a separate but coordinate way to produce the total moment at the steering wheel. The hands apply important moments on the steering wheel, not only forces. Referring to the rotation of the steering wheel, the bare moment produced by one hand is almost counteracting the moment produced by the tangential force produced by the same hand. The coordination of the different Neuro-Muscular Systems deserves a proper study. New driver models will possibly need to include specific NMS activation for each single arm and wrist and hand. The NMS of the driver model should consider reflex. The inclusion into new driver models of the activation of many different Neuro-Muscular Systems could in principle provide a better estimation of the driver's reaction time. The information provided by the early employment of the new ISW appears to be original and may open a possible field of research focused on the development of next generation driver models.

\section{Conflict of interest statement}

None of the authors have any commercial relationships which represent a past, present or future conflict of interest.

\section{Acknowledgements}

Dott. Ing. A Chiaramonte Radicella of Toyota Motor Italy is gratefully acknowledged for having supported the tests with Toyota Auris.

\section{Funding}

The Italian Ministry of Research has partially funded the presented research under the project ITS Italy 2020, grant CTN01_00176_166195.

\section{References}

[1] Mastinu G and Plöchl M, Road and off-road Vehicle System Dynamics Handbook, CRC Press, Boca Raton USA, 2014. ISBN 9781420004908.

[2] Perallos A, Hernandez-Jayo U, Onieva E, and García-Zuazola I J, Intelligent transport systems : technologies and applications, Wiley, 2016, ISBN 978-1-118-89478-1.

[3] Lu M, Wevers K, and Van Der Heijden R, Technical Feasibility of Advanced Driver Assistance Systems (ADAS) for Road Traffic Safety, Transportation Planning and Technology, 28:3, 167-187, 2005, https://doi.org/10.1080/03081060500120282.

[4] Atay F M, Complex time-delay systems, Theory and Applications, Springer Verlag, Berlin, 2010, ISBN 978-3-642-02328-6.

[5] Plöchl M and Edelmann J, "Driver models in automobile dynamics application," Vehicle System Dynamics, vol. 45, no. 7-8, pp. 699-741, Jul. 2007. https://doi.org/10.1080/00423110701432482.

[6] Cole D J, Occupant-vehicle dynamics and the role of the internal model, Vehicle System Dynamics, 56:5, 661-688, 2018, https://doi.org/10.1080/00423114.2017.1398342.

[7] Pick A J, "Neuromuscular Dynamics and the Vehicle Steering Task," Cambridge University, 2004.

[8] Pick A J and Cole D J, "Measurement of Driver Steering Torque Using Electromyography," Journal of Dynamic Systems, Measurement, and Control, vol. 128, no. 4, p. 960, Dec. 2006. http://dx.doi.org/10.1115/1.2363198 .

[Digitare qui] 
[9] Pick A J and Cole D J, "Dynamic properties of a driver's arms holding a steering wheel," in Proceedings of the Institution of Mechanical Engineers, Part D: Journal of Automobile Engineering, 2007, vol. 221, no. 12, pp. 1475-1486. https://doi.org/10.1243/09544070JAUTO460.

[10] Pick A J and Cole D J, "A Mathematical Model of Driver Steering Control Including Neuromuscular Dynamics," Journal of Dynamic Systems, Measurement, and Control, vol. 130, no. 3, p. 31004, May 2008. http://dx.doi.org/10.1115/1.2837452.

[11] McMulkin M L and Woldstad J C, "Effects of wheel design on the torques applied to large hand wheels," International Journal of Industrial Ergonomics, vol. 15, no. 3, pp. 205-213, Mar. 1995.

[12] "Kyowa Electronic Instruments Co., Ltd.," http://www.kyowa-ei.com/. [Accessed 14 January 2019].

[13] "PCB Piezotronics Inc," http://www.pcb.com. [Accessed 14 January 2019].

[14] "Rainer Thomas Messtechnik GmbH," https://www.rt-m.de/. [Accessed 14 January 2019].

[15] "ZSE Electronic GmbH," https://www.zse.de. [Accessed 14 January 2019].

[16] Gabrielli F, Pudlo P, and Djemai M, "Instrumented steering wheel for biomechanical measurements," Mechatronics, vol. 22, no. 5, pp. 639-650, Aug. 2012. https://doi.org/10.1016/j.mechatronics.2012.02.006.

[17] Hada M, Yasuda E I, Kobayashi T, Yamaguchi T, and Hattori Y, "Inverse Dynamics Analysis of DriverSeat-Steering System using Multibody Human Model," in Proceedings on ECCOMAS Thematic Conference on Multibody Dynamics, 2015.

[18] Kim J H and Song J B, "Control logic for an electric power steering system using assist motor," Mechatronics, vol. 12, no. 3, pp. 447-459, Apr. 2002.

https://doi.org/10.1016/S0957-4158(01)00004-6.

[19] Université de Valenciennes et du Hainaut-Cambrésis, "Le projet VOLHAND," http://www.univvalenciennes.fr/volhand/. [Accessed January 2017].

[20] Baronti F, Lenzi F, Roncella R, and Saletti R, "Distributed Sensor for Steering Wheel Grip Force Measurement in Driver Fatigue Detection," in Proceedings of the Conference on Design, Automation and Test in Europe. European Design and Automation Association, 2009, pp. 894-897. https://doi.org/10.1109/DATE.2009.5090790.

[21] Park J, "Plastic optical fiber sensor for measuring driver-gripping force," Optical Engineering, vol. 50, no. 2, p. 20501, Feb. 2011. https://doi.org/10.1117/1.3542040.

[22] Eksioglu M and Kızılaslan K, "Steering-wheel grip force characteristics of drivers as a function of gender, speed, and road condition," International Journal of Industrial Ergonomics, vol. 38, no. 3-4, pp. 354-361, Mar. 2008. https://doi.org/10.1016/j.ergon.2008.01.004.

[23] Garinei A and Marsili R, "Development of a new capacitive matrix for a steering wheel's pressure distribution measurement," International Journal of Industrial Ergonomics, vol. 44, no. 1, pp. 114119, Jan. 2014. https://doi.org/10.1016/j.ergon.2013.11.012.

[24] Abdul Rahim H, Yusop Z, and Abdul Rahim R, "Drowsy Driver Detection via Steering Wheel," Sensors \& Transducers Journal, vol. 120, no. 9, pp. 119-133, 2010.

[25] Lin $Y$, Leng $H$, Yang $G$, and Cai $H$, "An Intelligent Noninvasive Sensor for Driver Pulse Wave Measurement," IEEE Sensors Journal, vol. 7, no. 5, pp. 790-799, May 2007. https://doi.org/10.1109/JSEN.2007.894923.

[26] Haasnoot R A and Mansfield N J, "Effect of push/pull and grip force on perception of steering wheel vibration," Conference Proceedings 10th International Conference on Hand-Arm Vibration, pp. 287293, 2004.

[27] Neureder U, "Investigation into steering wheel nibble," in Proceedings of the Institution of Mechanical Engineers, Part D: Journal of Automobile Engineering, 2002, vol. 216, no. 4, pp. 267-277. https://doi.org/10.1243/0954407021529101.

[Digitare qui] 
[28] Ayglon V, Cherian V, and Jalili N, "Experimental Testing and Validation of Tangential Steering Wheel Vibrations Due to Tire Nonuniformity," in Dynamic Systems and Control, Parts A and B, 2005, vol. 2005, pp. 545-551. http://dx.doi.org/10.1115/IMECE2005-81594.

[29] Mastinu G, Gobbi M, and Previati G, "A New Six-axis Load Cell. Part I: Design," Experimental Mechanics, vol. 51, no. 3, pp. 373-388, Mar. 2011. https://doi.org/10.1007/s11340-010-9355-1.

[30] Mastinu G, Gobbi M, Device and method for measuring forces and moments, Patent US10565338.

[31] Mathworks, MATLAB User's Guide. Natik, MA, USA: The Mathworks, 2014.

[32] Young W C, Budynas R G, and Sadegh A M, Roark's formulas for stress and strain. McGraw-Hill, 2012. ISBN 9780071742474.

[33] Mastinu G, Gobbi M, Miano C, Optimal Design of Complex Mechanical Systems, with Application to Vehicle Engineering, Springer Verlag, Berlin, 2006.

[34] Dassault Systèmes, Abaqus analysis user's manual. Providence, RI, USA: Simulia Corp., 2007.

[35] “Omega," https://it.omega.com/. [Accessed 14 January 2019].

[36] "Saati S.p.A.," http://www.saati.com/. [Accessed 14 January 2019].

[37] Gibson R F, Principles of composite material mechanics. CRC Press, 2016. ISBN 9781439850053.

[38] Tsai S W and Wu E M, "A General Theory of Strength for Anisotropic Materials," Journal of Composite Materials, vol. 5, no. 1, pp. 58-80, Jan. 1971. https://doi.org/10.1177/002199837100500106.

[39] Narayanaswami R and Adelman H M, "Evaluation of the Tensor Polynomial and Hoffman Strength Theories for Composite Materials," Journal of Composite Materials, vol. 11, no. 4, pp. 366-377, Oct. 1977. https://doi.org/10.1177/002199837701100401.

[40] Antoniou A, Digital Signal Processing. McGraw Hill, 2016. ISBN 9780071846035.

[41] "Microchip Technology Inc.," http://www.microchip.com/.[Accessed 14 January 2019].

[42] "MTS," http://www.mts.com/. [Accessed 14 January 2019].

[43] Quarteroni A, Sacco R, and Saleri F, Matematica Numerica. Milano: Springer Milan, 2008. ISBN 9788847007826.

[45] "Toyota," https://www.toyota.com/. [Accessed 14 January 2019].

[46] "Kvaser," https://www.kvaser.com/. [Accessed 14 January 2019].

[47] "OxTS," http://www.oxts.com/. [Accessed 14 January 2019].

[48] “ACI-SARA LAINATE," http://www.vallelunga.it/it/aci-sara-lainate/.[Accessed 14 January 2019].

[49] Hada M, Yamaguchi T, and Hattori Y, "Visualization of driver's body motion by instantaneous screw axis and line of action," in Proceedings of 24th Symposium of the International Association for Vehicle System Dynamics (IAVSD 2015), 2016, pp. 799-808. http://dx.doi.org/10.11351/jsaeronbun.46.161.

[50] Comolli F, Ballo F M, Gobbi M, and Mastinu G, "Instrumented Steering Wheel: Accurate Experimental Characterisation of the Forces Exerted by the Driver Hands for Future Advanced Driver Assistance Systems". In ASME 2018 International Design Engineering Technical Conferences and Computers and Information in Engineering Conference. American Society of Mechanical Engineers. 2018, p. V003T01A006-V003T01A006. http://dx.doi.org/10.1115/DETC2018-85931. 